\title{
Expected endings and judged duration
}

\author{
MARI RIESS JONES \\ Ohio State University, Columbus, Ohio \\ MARILYN G. BOLTZ \\ Haverford College, Haverford, Pennsylvania \\ and \\ JAMES M. KLEIN \\ Ohio State University, Columbus, Ohio
}

\begin{abstract}
In four experiments, the predictions of an expectancy/contrast model (Jones \& Boltz, 1989) for judged duration were evaluated. In Experiments 1 and 2, listeners estimated the relative durations of auditory pattern pairs that varied in contextual phrasing and temporal contrast. The results showed that when the second pattern of a pair either seems to (Experiments 1 and 2) or actually does (Experiment 2) end earlier (later) than the first, subjects judge it as being relatively shorter (longer). In Experiment 3, listeners heard single patterns in which notes immediately preceding the final one were omitted. Timing of the final (target) tone was varied such that it was one beat early, on time, or one beat late. Listeners' ratings of target tones revealed systematic effects of phrasing and target timing. In Experiment 4, listeners temporally completed (extrapolated) sequences of Experiment 3 that were modified to exclude the target tone. The results again showed that phrase context systematically influenced expectancies about "when" sequences should end. As a set, these studies demonstrate the effects of event structure and anticipatory attending upon experienced duration and are discussed in terms of the expectancy/contrast model.
\end{abstract}

Why does one song on the radio seem longer than another when they are actually the same duration? This sort of temporal distortion is not uncommon. It corresponds to a phenomenon of special interest in the study of time estimation, known as the filled-interval effect, in which two equivalent time intervals are incorrectly judged as differing in total duration as a function of the information or activities that fill them. It figures in the development of many influential theories of duration judgment (see Allan, 1979; Block, 1979; Fraisse, 1978, 1984, 1987, for reviews).

In the present research, the filled-interval effect within the larger domain of attentional strategies and their impact upon experienced duration is reconsidered. In particular, using various time estimation tasks, we examined some ways in which the information that fills a time interval can systematically influence duration judgments. We pose the following question: "Does the temporal patterning of information within an event affect people's ex-

\footnotetext{
This research was partially supported by National Science Foundation Grant BNS-8403521, awarded to the first author. The authors are specially indebted to Wicher van Vreden, who participated in the design, data collection, and write-up of Experiment 1 and also to Jessica Eisenhaure for the collection of data in Experiment 2. In addition, we thank R. Block, A. Gabrielsson, J. Jackson, A. Kristofferson, R. Melara, J. Michon, R. Proctor, J. Ralston, and several anonymous reviewers for many helpful comments on earlier versions of this manuscript. Requests for reprints should be sent to $M$. R. Jones, Department of Psychology, Townshend Hall, The Ohio State University, Columbus, Ohio 43210.
}

pectancies about 'when' it will end, and hence 'how long' it seems to last?"

\section{Models of Time Estimation}

Models addressing the filled-interval effect share several underlying assumptions. The most fundamental of these posits that experienced duration depends on the amount of processing activity required by a given event. In particular, for many contexts it is assumed that the more complex of two, otherwise equivalent, events requires more "processing" and so will seem longer. Theoretical differences emerge with definitions of complexity and the cognitive referent for duration judgments. The latter may involve a memory store, as seen in Ornstein's storage size hypothesis (1969), adapted from Frankenhauser (1959), in which judged duration is assumed to increase with the number of "chunks" stored in memory. Or the referent may involve the amount of attentional effort invested during a given time interval such that greater attention can increase duration judgments (Thomas \& Weaver, 1975; Underwood \& Swain, 1973). Finally, time estimates may depend on the amount of change associated with an event's context, its cognitive processing, or the organismic variables within an individual (Block, 1979, 1985, 1989).

Although these approaches have generated a substantial amount of research, the literature is also characterized by divergent and conflicting results. With musical events, melodic complexity, as conventionally measured in terms of amount of change, and so on, appears to have no differential effects on duration judgments (e.g., Clarke 
\& Krumhansl, 1990; Jones \& Boltz, 1989). More generally, manipulations of stimulus familiarity (Avant \& Lyman, 1975, vs. Devane, 1974), stimulus arrangement (Schiffman \& Bobko, 1974, vs. Poynter, 1983), and task difficulty (Underwood \& Swain, 1973, vs. Burnside, 1971) indicate that more complex events are sometimes judged as being longer, but on other occasions as shorter than less complex events. Some, but not all, of these discrepancies may relate to task differences (e.g., prospective vs. retrospective) and whether or not a listener knows in advance that a time judgment will be required (e.g., see Brown, 1985)

These findings have led some researchers to suggest that assumptions motivating this research warrant reexamination. One proposal is that conflicting findings imply the operation of two cognitive mechanisms (e.g., a timer and a nontemporal processor) that differentially compete for attentional resources (e.g., Brown, 1985; Zakay, 1989). Others, such as Block $(1985,1989)$, emphasize that the overall contextual relationship between a perceiver and the environment affects judged duration. Finally, Jones and Boltz (1989) suggest that the patterning of nontemporal information within a time interval differentially affects attention and duration judgments; this resulted in the expectancy/contrast model.

\section{An Expectancy/Contrast Approach to Time Estimation}

According to this model, initially developed by Boltz (1985) and extended by Jones and Boltz (1989), environmental events vary in their degree of structural coherence, and this in turn influences people's mode of attending and time estimates. At one end of this structural continuum are highly coherent events that contain a nonarbitrary beginning and end, as well as a characteristic time span filled with temporally nested information. At the other end are events that lack coherence; in these, the temporal patterning of information is less regular. For example, musical events can display high coherence because compositional constraints determine melodic phrases that are often grouped (nested) into higher level phrases and themes. When such a composition is interpreted by experts, its coherence becomes evident because these nested phrasings clearly emerge in the produced time pattern. However, when played by novices, who typically falter and insert inappropriate (i.e., agrammatical) pauses, the resulting auditory event is less coherent (Palmer, 1989).

In the present research, we focused on relatively coherent events. In these, an event's nontemporal content (e.g., tones, words, actions) is arranged in time to form prominent groups and phrases. A group or phrase is prominent when its time span is redundantly marked by salient elements such as the joint occurrence of a special nontemporal marker with a distinctive temporal one (e.g. prolonged duration, or pause). In music, for example, beginnings and/or ends of phrases are often signaled by the cooccurrence of a salient pitch (e.g., the keynote), which is lengthened. Longer, tonally salient pitches emphasize phrasing by lending a sense of completeness or resolution to endings ${ }^{1}$ (Kramer, 1982; Meyer, 1956). In coherent events, prominent phrases are often recursively grouped (nested) in simple ways within larger phrases that have proportionately larger time spans (e.g., Lerdahl \& Jackendoff, 1983). In fact, when these temporal nestings realize simple proportions (e.g., doubling of time spans), they add to the event's coherence by virtue of forming simple time hierarchies (Boltz, 1991a, 1992b; Boltz \& Jones, 1986; Jones, 1987; Jones \& Ralston, 1991). Such arrangements are not unique to music. They also occur in speech utterances (e.g., Grosjean, Grosjean, \& Lane, 1979), locomotion patterns (e.g., Cutting, 1981; Gentner, 1987; Pierson, 1976), action sequences (e.g., Newtson, 1973), and narrative discourse (e.g., Mandler \& Johnson, 1977).

We assume that coherent events are most likely to afford a mode of attending, termed future oriented. In this mode, attending is influenced by an event's higher order time structure and occurs when simple, prominently marked time hierarchies are present in an unfolding event. These time relationships enable an attender to anticipate and extrapolate the event's future course. Attentional extrapolations amount to event-specific expectancies that permit an attender to anticipate the "what" and "when" of future changes, including the event's ending (Boltz, 1992a; Cooper \& Sorenson, 1977; Jones \& Boltz, 1989; Kramer, 1982; Scott, 1982; Todd, 1985).

According to this view, future-oriented attending can bias time estimates. People tend to judge how long an event seems according to how it confirms or violates expectancies about "when" it will end. Thus, let E stand for an event's expected ending time; $E$ is event-specific in that it depends on some abstraction of time relations within the preceding context. ${ }^{2}$ Violations of $E$ occur when the observed event ending $(\mathrm{O})$ either exceeds or falls short of $E$. These violations correspond to momentary surprises, termed temporal contrasts, and are formalized as $\mathrm{O}-\mathrm{E}$, the signed difference between expected and observed ending times. In terms of the filled-interval phenomenon, the gist of the model is this: If two time intervals both end as expected (i.e., on time), then they will be correctly judged as being equal in duration. However, if one seems to end earlier than expected (i.e., negative temporal contrast), then it will be judged as being shorter, whereas if it seems to end later than expected (i.e., positive temporal contrast), then it will be judged as being longer. These contrasts involve event endings. We assume that they bias overall time judgments because endings are salient delimiters of an event's total duration.

Events that lack coherence do not support futureoriented attending (Jones \& Boltz, 1989). In terms of their time structure, they fail to support temporally sustained event-specific expectancies. Instead, they encourage a mode of attending that is termed analytic attending. Analytic attending is influenced by lower time levels in which smaller event "details" occur (e.g., changes in pitch direction or sound qualities). In these cases, when infer- 
ring relative duration, people may simply count and compare the total number of chunks or changes, thus relying on strategies suggested by Ornstein (1969) or Block (1979, 1985,1989 ).

In sum, highly coherent events encourage a futureoriented mode of attending in which duration estimates will depend on temporal contrast arising from the confirmation/violation of expectancies. Conversely, less coherent events will result in an analytic mode of attending wherein people respond to an accumulation of local chunks and changes in estimating duration judgments.

\section{Empirical Support}

Most of the previous research on the filled-interval effect has relied on events of low coherence. In this respect, effects of analytic attending are well documented. By contrast, we know less about time estimates of coherent events, and hence future-oriented attending. One researcher who addressed this is Boltz (1989a, 1989b), who varied expectancy confirmation/disconfirmation in coherent folk tunes by manipulating their final pitches. Some final tones confirmed tonal expectancies about endings, whereas others did not. In the latter, the unresolved tunes left listeners "hanging"; their endings seemed early, and the tunes, as a whole, were underestimated in duration. In other experiments, Jones and Boltz (1989) used folk tunes in which they manipulated tune coherence and final phrase duration. The coherent tunes had phrase structures designed to promote future-oriented attending in that they approximated simple, prominently marked time hierarchies. Final phrase durations were varied such that in some tunes the last note seemed to occur "too early" (i.e., negative temporal contrast) or "too late" (i.e., positive temporal contrast) relative to preceding phrase periodicities. The results indicated that as coherence increased, under- and overestimations of tune durations became more dependent on, respectively, negative and positive temporal contrasts. This was true even though all the melodies were equivalent in total duration and amount of nontemporal information.

In the present research, additional predictions of the expectancy/contrast model were pursued in four experiments. In the first two studies, we tested predictions about temporal contrast effects on comparative time judgments. In these, the phrase structure of coherent events was manipulated to create expectancies and temporal contrasts (expectancy violations) of different directions and magnitudes. In the second two experiments, we relied on extrapolation and rating tasks to explore the assumption that expectancies are timed attentional extrapolations that are event specific.

\section{EXPERIMENT 1}

In Experiment 1, we addressed the generality of results previously reported by Jones and Boltz (1989). We relied on simpler, and largely coherent melodies and considered whether temporal contrast continues to influence time estimates when it arises from manipulations of ex- pectancy rather than from changes in final phrase duration. Temporal contrast is a difference between an expected and observed duration $(\mathrm{O}-\mathrm{E})$, and so its value depends upon both how long a listener expects (E) a final phrase to last and how long it actually lasts $(\mathrm{O})$. Jones and Boltz (1989) and Boltz (1991b) examined contrast effects as a function of variations in final phrase durations, $O$, while holding $E$ constant. Here, we manipulated contrast by varying $E$ and holding final phrase duration constant. This manipulation assumes that $\mathrm{E}$ depends on an event's phrasing structure (an assumption explored in Experiments 3 and 4 ).

Figure 1 illustrates how phrase context, and therefore expectancy, affects temporal contrast. Several coherently phrased sequences appear where each phrase consists of a series of low (beat marker) pitches and ends with a high (phrase marker) pitch. The beat marker pitch marks lower order time periods, termed beat periods; the phrase marker pitch, which is rhythmically lengthened, redundantly marks higher order time periods, termed phrase durations. For example, the sequence of Figure 1a has five marked phrases, each with eight beat periods. Assuming that the opening phrases induce an expectancy (E) for a final phrase of eight beats, this sequence neatly confirms it. Formally, E (measured in beat periods) is eight beats in length. When the observed final phrase, $O$, is also eight beats long, the resulting contrast, $c^{\prime}$ (i.e., $c^{\prime}=$ $\mathrm{O}-\mathrm{E}$ ) becomes a null contrast: $8-8=0$.

Figures $1 \mathrm{~b}$ and $1 \mathrm{c}$ indicate, respectively, the ways in which manipulations of phrase context can lead to nonzero temporal contrasts (with the same final phrase). For example, if $E$ is based on the opening phrases of 4 beats (e.g., Figure 1b), then a final deviant phrase of 8 beats leads to a temporal contrast, $c^{\prime}=+4$ beats. Similarly, a phrase context of 12 beats (Figure 1c) leads to a contrast value of $c^{\prime}=-4$ beats. In brief, the direction and magnitude of temporal contrast results from the way information is patterned to induce an expectancy, $\mathrm{E}$, while holding $\mathrm{O}$ constant.

In Experiment 1, we used various null $(\mathrm{N})$ and expectancy-based contrasts (E) that were derived from pairs of sequences such as those shown in Figure 1. The two sequences (standard and comparison) of a pair always lasted the same amount of time and contained the same amount of information, as indexed by numbers of tones, beats, phrases, chunks, and changes. Furthermore, both sequences were presented at the same relatively fast rate to discourge explicit counting strategies. Thus, sequences within a pair differed only with regard to phrasing that could yield a temporal contrast of a specified direction and magnitude.

Two tasks were posed for the listeners. First, a prospective comparative judgment task was used to assess temporal contrast effects; the listeners judged the duration of the comparison relative to its standard in each pair. If temporal contrast matters, then a comparison will be incorrectly judged as being shorter whenever it contains a negative contrast $\left(c^{\prime}<0\right)$, and longer whenever it contains a positive contrast $\left(c^{\prime}>0\right)$. 


\section{Examples of Temporal Contrast as a Function of Melodic Phrasing}

\section{a. ZERO CONTRAST}

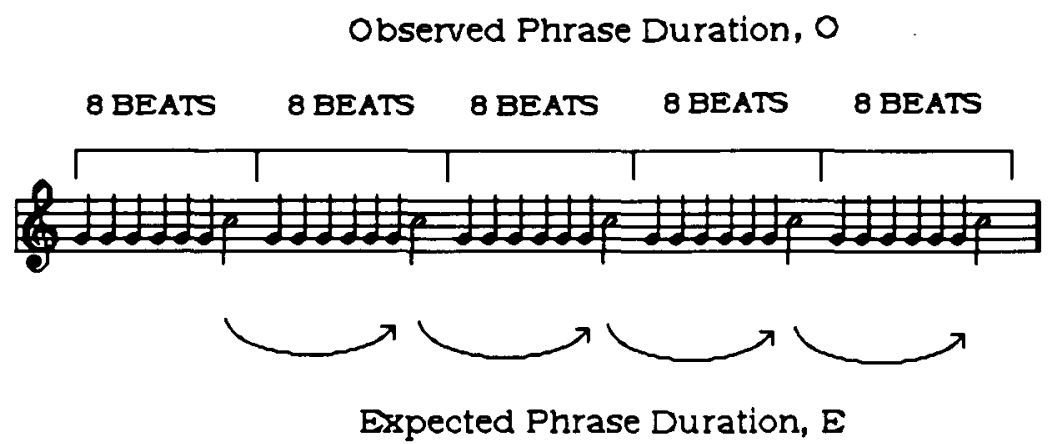

b. POSITTVE CONTRAST

Observed Phrase Duration, $\mathrm{O}$
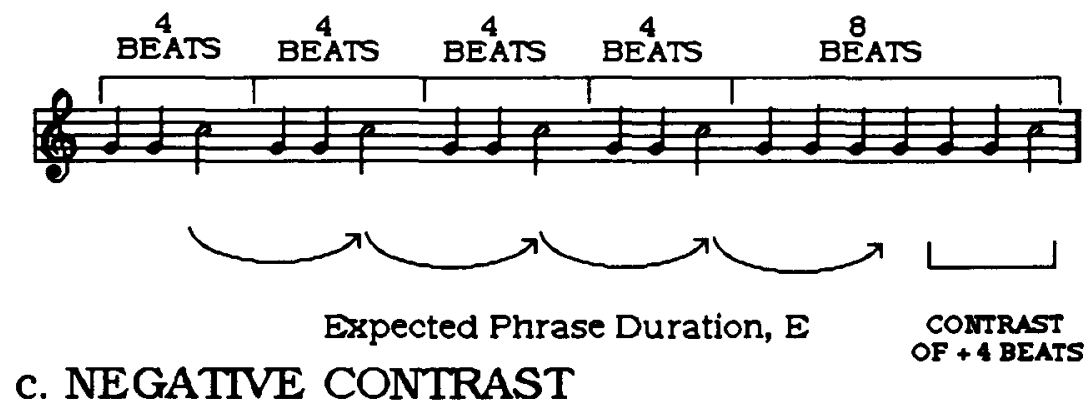

Observed Phrase Duration, 0
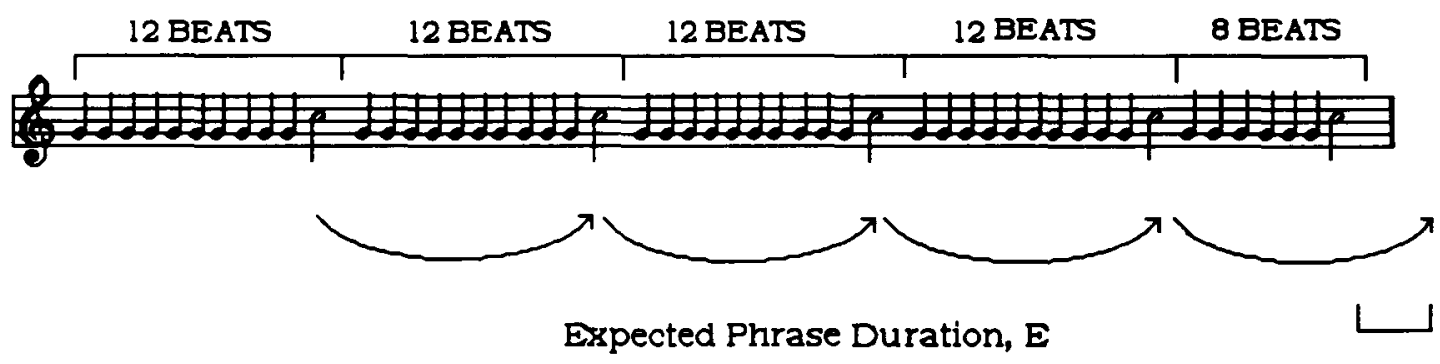

CONTRAST

OF - 4 BEATS

Figure 1. Three exemplar sequences from Experiment 1. In panel a, observed phrase durations (indicated by phrase markers) always recur after 8 beats, thus creating no contrast between observed and expected ending times. In panel b, observed phrase durations all last 4 beats, but the final phrase lasts 8 beats. This creates a positive contrast $\left(c^{\prime}=+4\right.$ beats). Conversely, panel $c$ illustrates a negative contrast $\left(c^{\prime}=-4\right.$ beats). Relative to preceding phrase durations, the final observed period ends 4 beats before its expected ending time. (Note-In these patterns, $O=1$ beat and $O=2$ beats.) 
Second, a production task (to each sequence of a pair), was used to encourage future-oriented attending and to assess event-specific expectancies (i.e., $\mathrm{E}$ alone). The listeners were asked to actively anticipate the onset of the last note for each sequence via a buttonpress. If people generate event-specific expectancies, then produced times should be positively related to the length of a pattern's context phrases. For example, people should anticipate earlier endings in a sequence with briefer phrasings (i.e., phrase contexts of 4 or 6 beats), and later endings for contexts based on longer phrasings (e.g., the 10- or 12-beat phrases). In other words, production times should be more sensitive to phrasing context and its influence on expectancies than to the observed duration of the final phrase and, hence, temporal contrast.

In sum, standard and comparison melodies of equivalent duration and amount of nontemporal information were presented to listeners for comparative time judgments and production responses. The expectancy/contrast approach predicts over- and underestimates as a function of the direction and magnitude of temporal contrasts. In addition, if production times reflect event-specific expectancies, then they should vary systematically with phrase context, rather than with temporal contrast values.

\section{Method}

\section{Design and Subjects}

The design was a $5 \times 2 \times 3 \times 2$ mixed factorial. Five levels of phrase context (phrase length, $\mathrm{L},=4,6,8,10,12$ beats) were crossed with two levels of context type (null, $\mathrm{N}$; expected, E), and three levels of melodic instance $(\mathrm{C}, \mathrm{G}$, or $\mathrm{F}$ as the beat pitch). Counterbalance order $(1,2)$ was the single between-subjects variable.

Twenty-six subjects from an introductory psychology course at The Ohio State University participated in the experiment for course credit. Each had moderate musical experience--at least 4 years of training within the past 6 years-and was currently playing a musical instrument.

\section{Stimulus Structure}

In all the conditions, three different melodic instances were constructed from two component pitches. The lower pitch (i.e., the beat marker) provided the sequence of beats within a pattern; it was either C4, G4, or F4 (where numbers refer to octaves). Phrase marker tones always occurred at phrase ending points and corresponded to a rise in pitch by four semitones (i.e., E4, B4, and A4). Beat marker periods were 220 msec (i.e., one beat, or l) in duration (onset-to-onset time) and consisted of a 200 -msec tone on time and a 20-msec off time. The phrase period level corresponded to the onset-to-onset time between phrase markers, and this time period varied according to the phrase context condition. In all the conditions, however, the phrase marker tone was longer than the beat marker tone, consisting of on and off times of 400 and $40 \mathrm{msec}$, respectively. Thus, the phrase marker time interval lasted two beats, or 2 . In all the conditions, the sequences contained five phrases

A total of 17 individual sequences varied according to phrase context and contrast type. They were combined to form 14 pairs of standard and comparison sequences, in which pair members were always equivalent in total duration (see Table 1 , left column). This set of stimuli consisted of 10 experimental pairs and 4 filler pairs.

Experimental pairs. Half of the experimental pairs realized a null $(\mathrm{N})$ contrast type and half an expected (E) contrast type. Null pairs are shown in Table 1 as $\mathrm{N1}, \mathrm{N} 2, \mathrm{~N} 3, \mathrm{~N} 4$, and N5, where phrase length, $L$, is, respectively, $L=4,6,8,10$, and 12 beat periods (beats). Both standard and comparisons had five identical phrases in null pairs. In $\mathrm{E}$ pairs, in order to manipulate contrast (holding total duration constant), a deviant phrase of $x$ beats began a standard sequence and ended its comparison sequence. The algorithm $x-n(\mathrm{~L})$ describes a standard pattern's phrasing as an initial deviant phrase, followed by $n(n=4)$ phrases of length L. For example, $8-4(4)$ was a standard sequence of successive phrase lengths of 8-4-4-4-4 beats. The algorithm was reversed for comparisons in E pairs: $n(\mathrm{~L})-x$ or $4(4)-8$. Thus, the E1 pair had standard and comparison sequences with phrase structures, respectively, of 8-4-4-4-4 and 4-4-4-4-8 beats.

The contrast parameter, $c^{\prime}$, indexes the direction and magnitude of a pairwise contrast. Thus, $c^{\prime}=0$ for all $\mathrm{N}$ pairs and $c^{\prime}=4$, $2,0,-2,-4$ beats for E1, E2, E3, E4, and E5 pairs, respectively. With the exception of $\mathrm{E} 3$, all $\mathrm{E}$ pairs yielded nonzero contrast values

Table 1

Mean Duration Judgments in Experiment 1 as a Function of Phrase Context and Contrast Type

\begin{tabular}{|c|c|c|c|}
\hline $\begin{array}{l}\text { Phrase } \\
\text { Context }\end{array}$ & Contrast & $\begin{array}{l}\text { Mean Judged } \\
\text { Duration }\end{array}$ & $\begin{array}{c}\text { Mean } \\
\text { Response Time }\end{array}$ \\
\hline \multicolumn{4}{|c|}{ Null Contrast } \\
\hline $\begin{array}{r}\text { N1 } 8-4(4) \\
8-4(4)\end{array}$ & 0 & 100.15 & +2.08 \\
\hline $\begin{array}{r}N 28-4(6) \\
8-4(6)\end{array}$ & 0 & 100.27 & +9.37 \\
\hline $\begin{array}{r}\text { N3 } 5(8) \\
5(8)\end{array}$ & 0 & 99.77 & -1.19 \\
\hline $\begin{array}{r}\text { N4 } 8-4(10) \\
8-4(10)\end{array}$ & 0 & 100.40 & +1.73 \\
\hline $\begin{array}{r}\text { N5 } 8-4(12) \\
8-4(12)\end{array}$ & 0 & 100.62 & -1.34 \\
\hline \multicolumn{4}{|c|}{ Expected Contrast } \\
\hline $\begin{array}{r}\text { E1 } 8-4(4) \\
4(4)-8\end{array}$ & +4 & 114.13 & -42.90 \\
\hline $\begin{array}{r}\text { E2 } 8-4(6) \\
4(6)-8\end{array}$ & +2 & 109.35 & -49.74 \\
\hline $\begin{array}{r}\text { E3 } 5(8) \\
5(8)\end{array}$ & 0 & 100.12 & -1.19 \\
\hline $\begin{array}{r}E 48-4(10) \\
4(10)-8\end{array}$ & -2 & 88.38 & +68.80 \\
\hline $\begin{array}{r}\text { E5 } 8-4(12) \\
4(12)-8\end{array}$ & -4 & 87.71 & +64.28 \\
\hline \multicolumn{4}{|c|}{ Filler Pairs } \\
\hline $\begin{array}{r}F 16-4(4) \\
4(4)-6\end{array}$ & +2 & 110.12 & -51.97 \\
\hline $\begin{array}{r}F 24-4(6) \\
4(6)-4\end{array}$ & -2 & 90.04 & +65.79 \\
\hline F3 $\begin{array}{r}10-4(8) \\
4(8)-10\end{array}$ & +2 & 107.98 & -39.63 \\
\hline F4 $\begin{array}{r}10-4(12) \\
4(12)-10\end{array}$ & -2 & 86.88 & +72.46 \\
\hline
\end{tabular}

Note-Column 1 shows standard/comparison sequence pairs generated from the algorithms of $x-n(\mathrm{~L}) / \mathrm{x}-n(\mathrm{~L})$ and $x-n(\mathrm{~L}) / n(\mathrm{~L})-x$ for null and expected contrast/filler pattern pairs, respectively (see Method section). Column 2 shows the signed magnitude of each contrast. Column 3 depicts mean judged duration in the comparative judgment task (values of 100 indicate equivalent relative duration; values $<100$ and $>100$ indicate, respectively, relative under- and overestimations). Column 4 depicts mean produced response times (in milliseconds); negative values reflect anticipatory behavior in which synchronization responses occurred before the actual ending of a comparison pattern, and positive values reflect responses occurring after the actual ending tone. 
associated with expectancy violations by the final 8-beat phrase. The E3 pair reflects a null contrast similar to N3.

Filler pairs. Also shown in Table 1 are the four filler pairs. The fillers were included to provide diversity and to prevent the listeners from discovering that certain experimental pairs violated expectancies in a special way-namely, with an 8 -beat final phrase. Thus, the filler pairs provided deviant final phrases of varying lengths, that is, $x=6,4$, and 10 beats.

The 14 sequence pairs were randomized into three blocks of 14 trials each. Each pair occurred a total of three times, once in each of the three pitch instantiations (C4, G4, F4), yielding a total of 42 trials per session. Blocks were arranged into two counterbalance orders $(1,2)$.

\begin{abstract}
Apparatus
All the sequences consisted of square wave tones that sounded "flutelike." They were generated by a Wavetek Model 159 waveform generator that was controlled by a PDP-11 microcomputer. In generation, a custom-built envelope generator imposed $10-\mathrm{msec}$ rise and fall times on all tones. Tonal frequencies were taken from the equally tempered scale of international pitch ( $A 4=440 \mathrm{~Hz}$ ) and were subjectively equated for loudness in the actual experimental context, according to the reports of two judges. Sequences of tones programmed by the microcomputer were presented on line through $\mathrm{AKG} / \mathrm{K} 240$ headphones to the listeners in a sound-attenuated room.
\end{abstract}

\section{Procedure}

A trial consisted of two melodic sequences-a standard followed by a comparison sequence. A 1-sec, high-pitched warning tone $(5 \mathrm{kHz})$ preceded each pattern of a pair, and the offset of the standard sequence was separated from the onset of the second warning tone by a $3-\mathrm{sec}$ period of silence. The subjects were asked to indicate, via a hand-held button response, when the onset of the final note within each sequence would occur. They were told to use a pattern's entire structure to predict this ending time, much as a performer might when playing in synchrony with others. The subjects' responses were automatically recorded by the computer.

After the presentation of each standard/comparison pair, a computer-controlled light signaled a subject to judge the comparison sequence relative to its standard. A response sheet was provided, which contained two horizontal lines marked with percentage points in which relative line lengths were constant over trials. The subjects were told that the top line length represented the standard pattern's total duration (designated $100 \%$ ) and that they were to mark the second line (containing values up to $200 \%$ ) to reflect the relative total duration of the comparison sequence as a proportion of the standard. They were allowed $5 \mathrm{sec}$ to make a response, after which time the next trial was signaled. The subjects were asked to remove their watches prior to an experimental session, and were tested in groups of 2-4 individuals.

\section{Results and Discussion}

Because melodic instance and counterbalance order contributed negligible effects $(F<1.00)$, mean ratings were collapsed over these variables. A two-factor, repeated measures analysis of variance (ANOVA) assessed the effects due to phrase context and contrast type. Two dependent measures were analyzed for each subject: a comparative duration judgment and a produced response time associated with the predicted ending of the comparison sequence. Each is discussed below.

\section{Comparative Duration Judgments}

The third column of Table 1 depicts mean judged duration as a function of phrase context and contrast type.
A significant interaction between these two variables revealed that temporal contrast within melodic pairs exerted a marked impact upon relative duration judgments $\left[F(4,96)=74.01, M S_{\mathrm{e}}=25.84, p<.001\right]$. As seen in Table 1 , null pairs were not judged as significantly differing from a value of 100 , indicating that these sequences were correctly judged as being equivalent in duration. However, systematic over- and underestimations occurred within sequences that displayed contrasts $\left(c^{\prime}\right)$ based on expectancy violations (E pairs). For example, the comparison patterns of $E 1$ and E2 pairs seemed to end later than expected (i.e., positive $c^{\prime}$ ), and both were overestimated relative to a value of $100 \%$ (for both, Tukey's post hoc comparisons yielded $p<.01$ ). On the other hand, the final endings of E4 and E5 pairs were constructed so as to seem relatively "early" (i.e., negative $c^{\prime}$ ), and these prompted underestimations of their relative durations ( $p<.01$ for both comparisons). The absolute degree of bias in these four conditions ranged from $9.35 \%$ to $14.13 \%$. Last, the E3 pair (included as a control) was judged to have equivalent total duration; the final 8-beat phrase of this comparison corresponded to the sequence's modal phrase duration, and hence it did not violate expectancies about ending times.

These results complement and extend the findings of Jones and Boltz (1989) and Boltz (199lb). In order to study the filled-interval effect, the to-be-judged intervals must be identical in total duration. To satisfy this constraint in investigations of temporal contrast, either expectancies based on phrase context must be held constant while contrast is varied with final phrase length (Boltz, 1991b; Jones \& Boltz, 1989), or final phrase length must be held constant while contrast is varied by changing context-based expectancies (the present study). The present findings confirm that both kinds of manipulations produce a similar pattern of results.

These data are consistent with the hypothesis that direction (positive vs. negative) of a temporal contrast affects time estimates. They are less clear with respect to contrast magnitude. Significant effects associated with the absolute value of $c^{\prime}$ appear only with positive contrasts. For example, the E1 pair containing a positive contrast of 4 beats had a greater impact than that of the E2 with a +2beat contrast $(p<.01)$. However, this effect did not maintain for negative contrasts (e.g., E4 vs. E5). We return to this point in Experiment 2.

Responses to filler pattern pairs (Table 1) revealed the same pattern of results that was found with the experimental pairs. Because these pairs displayed temporal contrasts that did not arise from a final 8-beat phrase, these findings illustrate a generality of effects due to event-specific expectancies.

Finally, these data speak to the role of amount of processing activity on time estimation. A common finding with retrospective time judgments is that duration estimates increase with the amount of information filling an interval (Block, 1985; Ornstein, 1969). On the other hand, in some prospective designs, time estimates decrease with more nontemporal information (Hicks, Miller, \& Kins- 
bourne, 1976; McClain, 1983; Zakay, Nitzan, \& Glicksohn, 1983). In the present design, which is a prospective one, the standard and comparison intervals in all pairs ( $\mathrm{E}$ and $\mathrm{N}$ ) contained identical amounts of information, leading to the prediction of null differences. Instead, both over and underestimations of the standard occurred in $\mathrm{E}$ pairs. As in Jones and Boltz (1989), the magnitude and direction of bias in these estimates were directly related to the magnitude and sign of temporal contrast. Of course, it remains possible that some contrasts require "more intensive" processing than others, but the nature of such processing is unclear.

\section{Production Responses}

The purpose of this task was to determine whether people generate event-specific expectancies such that produced times reflect the preceding phrase context of a pattern. A preliminary analysis of responses to standard sequences suggests that this is the case. These produced ending times did not significantly differ from true ending times or from the times produced to the subsequent comparison sequence $(F<1)$. The latter findings are especially interesting because they indicate, as predicted, that produced times in comparison sequences are affected primarily by phrase context, even when the actual duration of the final phrase, and hence temporal contrast, is varied.

Mean produced times to comparison sequences are shown in Table 1; negative values indicate anticipatory responses, which occurred before the actual ending of a sequence; positive values indicate delayed responses, which occurred after the end of the sequence. An overall ANOVA of these data revealed a significant interaction between phrase context and contrast type $[F(4,96)=4.34$, $\left.M S_{\mathrm{e}}=1,060.31, p<.003\right]$. A set of Tukey's post hoc comparisons $(p<.01)$ confirmed that average response times for $\mathrm{N}$ pairs continued to be highly accurate (i.e., they did not differ from zero), but those for E pairs systematically deviated from zero in interesting ways. With short contextual phrasing (i.e., E1, E2), response times were anticipatory and significantly less than zero; the reverse was true with longer phrases (i.e., E4, E5). Because final phrase duration in these sequences was identical (eight beats), these findings imply that listeners' expectancies about "when" patterns should end are determined by the preceding phrase context and not by temporal contrast.

To sum up Experiment 1, comparative duration judgments and production times systematically differed as a function of the temporal arrangement of pitches that filled to-be-judged intervals. These findings are inconsistent with approaches that assume that time estimates depend on amount of information, and are more consistent with an approach that addresses the patterning of information filling the to-be-judged intervals.

\section{EXPERIMENT 2}

Experiment 2 was designed to assess whether the results of Experiment 1 were due to biasing properties of temporal contrast, as predicted, or whether they stemmed from an alternative interpretation of those data. That is, the same findings could arise if listeners ignored the phrase context of both standard and comparison sequences and simply compared beat counts within their respective final phrases. Several aspects of the previous design argue against such an interpretation-successive tones occurred at rates that were not optimal for counting; the listeners were carefully instructed to judge total duration; and finally, the pattern of produced times suggests strongly that the listeners did not ignore the phrases preceding the final one. Nevertheless, it remains important to rule out this alternative explanation. Accordingly, standard and comparison pairs in Experiment 2 were designed with this in mind.

Three different kinds of final contrasts were designed for use in a "pure" comparative duration task (i.e., unaccompanied by production responses). In addition to null $(N)$ and expected (E) contrast pairs, we included a third type of pairwise contrast, a true contrast (T). The true contrast generalized the contrast manipulation to true time differences between standard and comparison sequences. The standard sequences of $T$ pairs were identical to those of $\mathrm{N}$ and $\mathrm{E}$ pairs, but their comparisons differed in that $T$ comparisons always contained a final phrase duration identical to that of its standard in duration and number of tones. If listeners do compare only final phrases of standard and comparison melodies, then sequences forming $\mathrm{T}$ pairs should be judged as being identical (i.e., they will function as $\mathbf{N}$ pairs). On the other hand, if listeners respond to phrase context and total durational differences, then they should correctly judge members of $T$ pairs as being different. The structure of $\mathrm{N}, \mathrm{E}$, and $\mathrm{T}$ pairs is shown in Table 2.

The sequences in Experiment 2 were similar to those of Experiment 1, but were longer (see Table 2). Longer melodies should not only discourage counting, but also enhance listeners' reliance on final phrase differences if this is a tempting strategy. In all other respects, tunes across the two experiments were comparable. A final control in Experiment 2 concerned the larger experimental context. In a session, the listeners encountered roughly equal numbers of $\mathrm{N}, \mathrm{E}$, and $\mathrm{T}$ pairs, thus providing no general basis for anticipating a particular ending for any comparison sequence. Design constraints ensured that the listeners could not know whether a comparison was from a $T$ or an $E$ pair until it ended.

In sum, Experiment 2 expanded the range and type of to-be-judged events. If listeners make comparative time judgments by ignoring the phrasing context of both melodies in a pair and simply focusing on their final phrases, then we expect not only to replicate Experiment 1 findings with $\mathrm{N}$ and $\mathrm{E}$ pairs, but also to find that sequences of $T$ pairs will be incorrectly judged as being equal in duration.

\footnotetext{
Method

Design and Subjects

The design was a $5 \times 3 \times 2 \times 4$ mixed factorial. The subjects received a series of sequence pairs that varied as a function of phrase
} 
Table 2

Pairs of Experimental and Filler Pitch Sequences Used in Experiment 2 for Each Condition of Phrase Context and Contrast Type

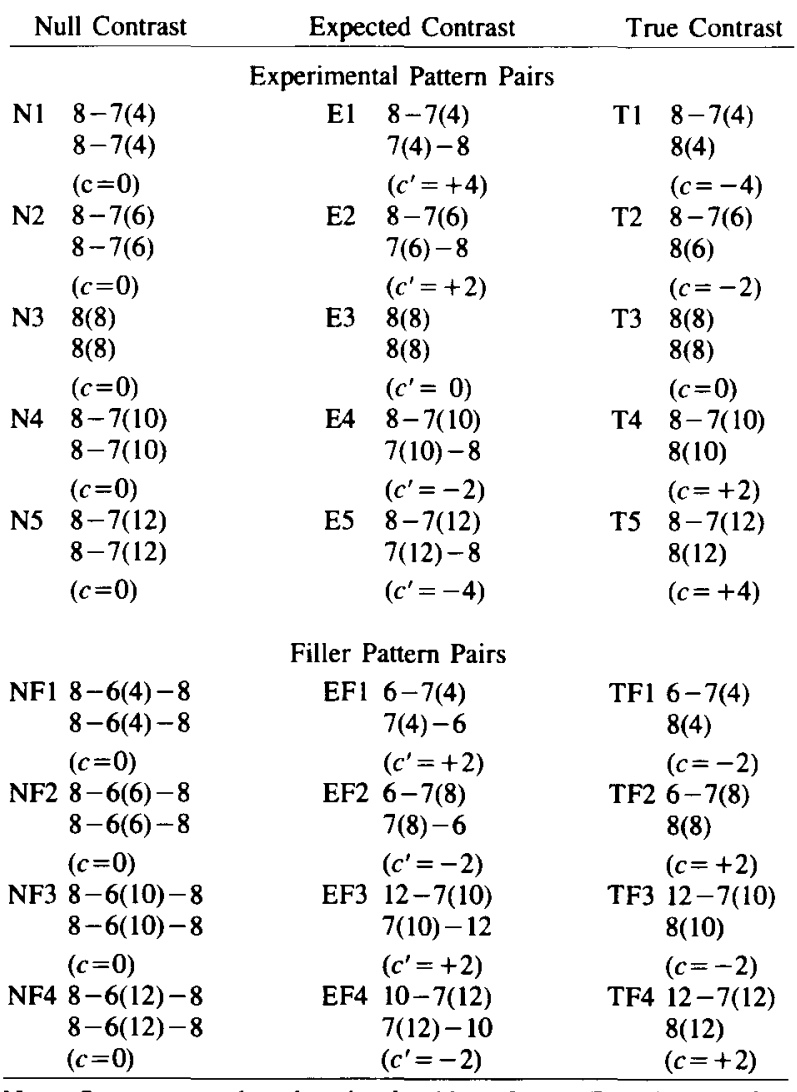

Note-Sequences are based on the algorithm of $x-n(\mathrm{~L})$, where $x$ refers to the number of beat periods in the initial deviant phrase and $n(\mathrm{~L})$ refers to the number $(n)$ of remaining phrases of a given length $(\mathrm{L})$. This algorithm is reversed for comparison patterns in the expected contrast condition, where the deviant phrase occurs last in a sequence, and it simplifies to $8(\mathrm{~L})$ for comparison patterns in the true contrast condition. Contrast values are indicated in parentheses.

context (phrase length, $\mathrm{L}=4,6,8,10$, or 12 beats), contrast type (null, expected, true), and starting pitch $(\mathrm{C} 4, \mathrm{G} 4)$. Counterbalance order $(1,2,3,4)$ was the single between-subjects variable.

Twenty-four subjects from an introductory psychology course at Haverford College participated in the experiment for course credit. Each had normal hearing, at least 4 years of musical training, and was currently playing an instrument. Each subject was randomly assigned to one of four counterbalance orders $(n=6)$.

\section{Stimulus Materials}

A total of 27 different pairs (12 experimental and 15 filler) of standard and comparison melodies were constructed (see Table 2). For a given phrase length, experimental pairs preserved the same standard pattern for $\mathrm{N}, \mathrm{E}$, and $\mathrm{T}$ contrasts; filler pairs did not necessarily observe this constraint. Both experimental and filler pairs consisted of two eight-phrase sequences, in which one phrase could deviate in length from the others. Again, the lengths of the deviant and other context phrases were denoted, respectively, by $x$ and $\mathrm{L}$ (beats). Together, experimental and filler pairs realized three types of contrasts: null contrast $(\mathrm{N})$, expected contrast $(\mathrm{E})$, and true contrast (T).

Experimental pairs. These pairs were constructed from the following rules for phrase context and contrast type manipulations:
Phrase context. Both sequences of experimental pairs contained eight phrases, and at least one of these was a deviant phrase of length $x=8$ beats. The remaining $n(=7)$ context phrases were all of length $L(L=4,6,8,10$, or 12 beats).

Contrast type. A given standard/comparison combination determined one of three contrast types: $\mathrm{N}, \mathrm{E}$, or T, all based on a common standard. As in Experiment 1, standard sequences followed the algorithm $x-n(\mathrm{~L})$. Thus, if $\mathrm{L}=4$ beats, then $8-7(4)$ indicates a standard pattern with phrasing 8-4-4-4-4-4-4-4. Contrast type depended on the presence and location of a comparison's deviant phrase.

Null contrast $(\mathrm{N})$ experimental pairs locate the deviant phrase ( $x=8$ beats) initially in both comparison and the standard. Thus, the standard/comparison pair, N1, has the formula $8-7(4)$ / $8-7(4)$, indicating that both open with an 8-beat phrase and continue with seven 4-beat phrases. This yields a zero, or null contrast.

Expected contrast (E) experiment pairs locate the deviant phrase initially in the standard, but finally in the comparison. Thus, the algorithm $x-n(\mathrm{~L}) / n(\mathrm{~L})-x$ becomes $8-7(4) / 7(4)-8$ for the E1 standard/comparison pair having $L=4$. Over different phrase contexts, E pairs determine expected contrasts, $c^{\prime}=4,2,0,-2,-4$ beats for E1, E2, E3, E4, and E5, respectively.

True contrast (T) experimental pairs enlist comparison patterns with no deviant phrase; the comparison algorithm simplifies to $8(\mathrm{~L})$, where $\mathrm{L}=4,6,8,10,12$ beats. For example, the $\mathrm{T} 1$ pair is denoted as $8-7(4) / 8(4)$, which yields a true total time difference of -4 beats. The pairs in Table 3 (T1, T2, T3, T4, T5) realize, respectively, five different true total time differences $(c)$ between standard and comparisons ( $c=-4,-2,0,2,4$ beats).

Filler pairs. These pairs were designed to prevent the listeners from engaging in certain probabilistic strategies that could arise from design constraints on the experimental pairs (see Table 3). Some filler pairs introduced comparisons that possessed final phrases of eight beats, but for which no expected contrast was evident (i.e., filler nulls). Others incorporated standard patterns that did not open with phrases of eight beats (filler $E$ and $T$ patterns). Finally, filler E pairs relied on comparisons in which the final phrase was not necessarily composed of eight beats (but that, nonetheless, yielded an expected contrast).

Standard-comparison pair trial blocks. The presentation of a sequence pair constituted a trial. Four counterbalance orders of 36 trials controlled for presentations of the two melodic instance types and two arrangements of experimental and filler pairs. Melodic instances were counterbalanced such that, in each counterbalance order, half the instances had start pitches of $\mathrm{C} 4$ and the rest had start pitches of G4. Conditions were counterbalanced such that each of the experimental pairs (null, expected, and true) appeared once, with the exception of the E3, N3, and T3 pairs, which each occurred twice. In addition, the $\mathrm{E} 2, \mathrm{~N} 2$, and $\mathrm{T} 2$ pairs also appeared twice (a noncritical error) to yield a total of 21 experimental trials. The remaining 15 trials in each order consisted of filler pairs in which each contrast type (N, E, T) occurred once, with the exception of NF1, EF1, and TF1, which each occurred twice.

Relative frequencies of experimental and filler pairs were carefully controlled to ensure that the listeners could not artifactually determine when and what contrasts might appear in various experimental pairs. These probabilities appear in Table 3 .

\section{Apparatus}

All the pattern sequences were constructed with the MIDILAB software system (Todd, Boltz, \& Jones, 1989), and were generated by a Yamaha TX81Z tone generator that was controlled by an IBM-AT computer with a Roland MPU-401 MIDI interface unit. Tone sequences were amplified by a Kenwood KR-A4010 receiver and recorded on a Nakamichi LX-3 cassette recorder. During an experimental session, blocks of prerecorded pattern pairs were presented on a JVC TD-V621 cassette player through Koss Pro 4AAA 
Table 3

Probabilities Associated With the Pitch Sequences of Experiment 2

Marginal probability

-of any change within a final phrase of a comparison pattern

-of a null $(c=0)$ contrast

44

.41

-of a true $(c=0)$ contrast

.25

-of an expected $\left(c^{\prime}=0\right)$ contrast

Conditional probability

- of a change in the final phrase length of a comparison pattern, given a null contrast pair

-of any change within a final comparison phrase, given $T$ and $\mathrm{E}$ pattern pairs (experimental plus fillers)

-of a final eight-beat phrase, given a comparison pattern that opens with a non-eight-beat phrase

-of a final comparison phrase of eight beats, given a standard pattern that opens with a phrase of eight beats

- of a $T$ or $E$ contrast for a particular kind of experimental standard

- of a true (nonzero) contrast and an expected (nonzero) contrast associated with a given experimental standard is the same

Note-Overall (marginal) probabilities are based on relative frequencies of certain pattern properties within the set of 36 pattern pairs; conditional probabilities are based on relative frequencies of other pattern features within specified subsets of the 36 pairs.

Plus headphones at a comfortable listening level. The timbre of all the melodies approximated that of a flute.

\section{Procedure}

On each trial, a 1-sec warning tone (pitch of E8) preceded the standard melody of a pair by 2 sec. Three seconds later, a compar- ison sequence occurred. The subjects were instructed to compare the total duration of the comparison sequence with its standard and to indicate this judgment on a response sheet containing two horizontal lines. They were told that the length of the top line represented the total duration of the standard sequence. The second line, directly underneath but indented halfway relative to the first line, was marked with percentile scores, ranging from $-50 \%$ to $+50 \%$. The subjects marked the second line to reflect a comparison's total duration as a proportion of the standard. In all other respects, the procedure paralleled that of Experiment 1.

\section{Results and Discussion}

The mean duration judgments of the experimental pairs are shown in Figure 2 as a function of contrast type $(\mathrm{N}$, $\mathrm{E}, \mathrm{T})$ and contrast value $\left(c, c^{\prime}\right)$. These data are collapsed over different phrase contexts to illustrate commonalities as a function of direction and magnitude of contrast. They are also collapsed over counterbalance order and starting pitch $(F<1.00$ for each). We consider performance with experimental pairs first; a second analysis considers filler pairs.

\section{Duration Judgments of Experimental Patterns}

An overall ANOVA indicated that the predicted interaction between contrast type and phrase context was large and significant $\left[F(8,160)=54.48, M S_{\mathrm{e}}=70.36, p<\right.$ $.001]$. Neither contrast type nor phrase context produced significant main effects. The interaction is explained entirely by contrast manipulations. As seen in Figure 2, the listeners correctly judged intervals within all null contrast pairs (averaged over $\mathrm{N}$ pairs with $c^{\prime}=0$ ) as being identical and those within $T$ pairs as being different. How-

\section{EXPERIMENT TWO JUDGED DURATION}

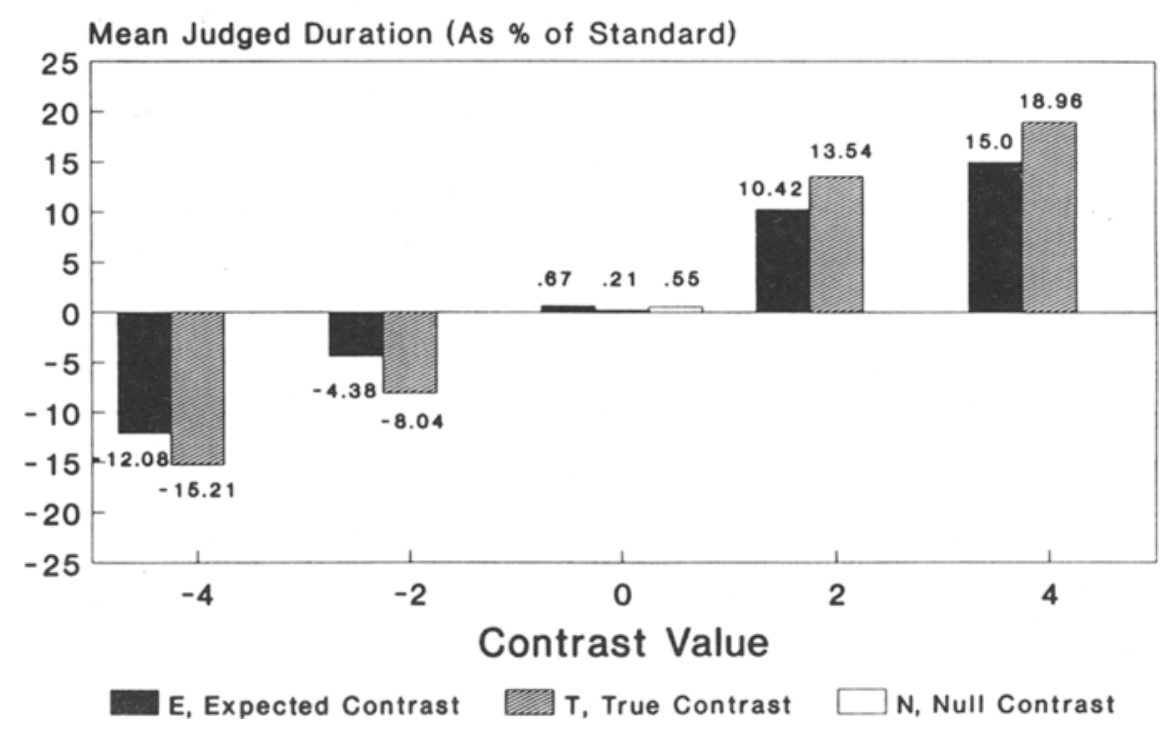

Figure 2. Mean judged duration as a percentage of the standard sequence's duration for expected, true, and null contrasts as a function of magnitude and direction of contrast in Experiment 2. The null contrast mean is averaged over all phrase lengths. 
ever, they incorrectly judged $\mathrm{E}$ pairs as differing in total duration.

First, consider what happens when two intervals really are different in total duration. When a $T$ comparison is shorter than its standard (i.e., $c<0$ ), listeners reliably judge this to be the case; similarly longer comparisons (i.e., $c>0$ ) are correctly judged as being longer. Standard and comparison sequences in $T$ pairs had final phrases that were identical in both duration and number of tones/beat periods, and because of this, such findings argue against the idea that people adopt a strategy of ignoring context and comparing isolated final phrases. Buttressing the contrast interpretation is the finding that here the listeners' ratings of the relative magnitude of durational difference increased directly with the magnitude of the true total time differences ( $c$ values) within T pairs. Comparisons that were only two beats shorter (or longer) than their respective standards produced significantly lower estimates than those with a four-beat difference. The reliability of these context effects were confirmed by a set of Bonferroni post hoc comparisons $(p<.01)$. In general, performance with $\mathrm{T}$ pairs demonstrated that the listeners did not respond simply to final phrases, but to true time differences involving total event durations.

From the perspective of the filled-interval effect, the more interesting questions concern expectancy-based temporal contrasts. As in Experiment 1, the comparison intervals of $E$ pairs were filled with the same amount of information as their standards, but were arranged to induce expectancies that could be subsequently violated. As shown in Figure 2, results with E pairs were remarkably similar to those with $\mathrm{T}$ pairs having comparable contrast values (i.e., where $c^{\prime}=c$ ). For example, E sequences that seemed to end "too early" [e.g., $c^{\prime}=-2$ beats from $8-7(10) / 7(10)-8$ ] were underestimated much as were $\mathrm{T}$ comparisons that really did end early [e.g., $c=-2$ beats from $8-7(6) / 8(6)]$. Parallel findings were obtained with positive contrasts. The magnitude of an expectancybased contrast also significantly affected time judgments of $E$ pairs in Experiment 2. Here, regardless of the direction of a contrast, E pairs with larger absolute contrast values (i.e., $\left|c^{\prime}\right|=4$ beats) yielded significantly higher ratings than those with smaller absolute values (i.e., $\left|c^{\prime}\right|$ $=2$ beats). A set of Bonferroni comparisons indicated that all magnitude differences among these pairs were significant $(p<.01)$.

In light of the parallel findings for $\mathrm{T}$ and $\mathrm{E}$ pairs, it appears that expectancy violations within E pairs "fool" people into thinking that a true difference exists between two events. That the listeners responded comparably to true time differences and to ones based on expectancies places the contrast values $c$ and $c^{\prime}$ on a similar footing. These findings not only suggest that the filled-interval effect is sensitive to expectancies and their violations, but that listeners may generally rely on temporal contrast to judge time differences (cf. Kristofferson, 1977).

Additional evidence for this conclusion can be seen from the ratings of $\mathrm{E}$ and $\mathrm{T}$ pairs that shared the same standard and differed only with respect to the final phrase length of their comparison sequences. In all of these pairs, the $T$ and $E$ contrasts were of equal magnitude but opposite signs: $c=-c^{\prime}$. Figure 2 indicates that in all cases the corresponding duration ratings of these $\mathrm{T}$ and $\mathrm{E}$ pairs were of equal magnitude and opposite in sign. For example, when the common standard of $T$ and $E$ pairs was 8-7(4), the $T$ comparison, $8(4)$, produced a true negative contrast of $c=-4$ beats and received a mean duration rating of -15.21 , whereas the E comparison, $7(4)$, for this standard produced a positive contrast of $c^{\prime}=+4$ beats and received a rating of +15.00 . In interpreting these data, it is important to emphasize that the design precludes that the listeners would intuit different $\mathrm{T}$ or $\mathrm{E}$ comparison endings in advance; surprise values of these endings were ensured because the conditional probability of a final deviant phrase was .50 (Table 3). Thus, it seems implausible that the listeners used a strategy that was based on comparing information within isolated final phrases of $E$ pairs and a different strategy, based on total durations, with $\mathrm{T}$ pairs. It is more likely that they responded in a similar manner to both $E$ and $T$ sequences. We suggest that their duration judgments reflect a general sensitivity to momentary violations of a contextually determined expectancy.

Finally, the above results cast doubt on the use of a simple counting strategy. For instance, such a strategy would require keeping track of rather complex phrase placements and counts of many tones (e.g., 150 in sequence pairs with 12-beat phrases). That is, if the listeners simply counted all the tones, their judgments would always be accurate with both $E$ and $T$ pairs, but this did not occur. On the other hand, if they counted only final phrase tones, then they would display the time distortions evident with $\mathrm{E}$ pairs, but would incorrectly judge $T$ pairs; this too did not occur. Finally, in addition to sequence length and structure, the tone rates here also discouraged efficient counting, at least by some accounts (e.g., Garner, 1951). Although Massaro (1976) reported that people can count relatively short sequences (up to eight tones) at rates this fast, his findings derive from listeners trained with over 2,000 such sequences (across a 5-day period).

\section{Duration Judgments of Experimental \\ Versus Filler Patterns}

The filler patterns were similar to the experimental ones, but they introduced uncertainty about the final phrase. Often they contained deviant phrases that were not eight beats in length. Thus, they provided an opportunity to assess the generality of effects observed in the previous analyses.

In general, the filler pairs produced a profile of judgments indistinguishable from that suggested in Figure 2. An overall ANOVA revealed a significant interaction of phrase context and contrast type that was not qualified by pattern type (experimental vs. filler) $[F(16,320)=$ $\left.40.13, M S_{\mathrm{e}}=71.69, p<.001\right]$. As with the experimental pairs, this interaction had two general features. First, the patterns in $\mathrm{N}$ pairs were judged as being equivalent 
in total duration. Second, the pattern pairs containing true or expected contrasts were judged as differing in total duration, with the direction of rated difference directly related to the sign of the true $(c)$ or expected $\left(c^{\prime}\right)$ contrast. In the true contrast condition, filler comparison patterns that were actually longer or shorter than their preceding standard were correctly judged to be so. Similarly, filler E pairs yielded relative under- and overestimations when comparison melodies ended earlier or later than expected (i.e., given the preceding context). These differences were confirmed by a set of Bonferroni post hoc comparisons $(p<.01)$. In sum, the effects observed in this experiment generalize to a more diverse set of melodies.

To sum up Experiment 2, the listeners' comparative time judgments indicate a sensitivity to null, expected, and true contrast differences. The judged time differences between standard and comparisons were reliably related to both the magnitude and direction of temporal contrast ( $c$ or $c^{\prime}$ ). Because listeners cannot predict and do not expect the final temporal contrast of a pattern, these data imply that when a temporal "surprise" does occur, it biases an individual's judgment about total duration.

\section{EXPERIMENT 3}

In Experiment 3, the construct of expectancy that underlies temporal contrast was examined. The expectancy/contrast model assumes that expectancies are (1) attentional extrapolations sustained over substantial time spans, and (2) event specific, determined by preceding context. In this experiment, we considered whether such expectancies obtain with listeners who have minimal formal musical training.

To evaluate the first assumption, the listeners were presented with individual sequences that contained a substantial silence in their final phrases. The length of the silent period was somewhat unpredictable in that it could occur after either $n$ (fewer) or $n+1$ (more) beat marker tones and was terminated by the final phrase tone (the target), which could occur early, on-time, or late (Figure 3). Nevertheless, if expectancies involve sustained extrapolations of attending based on prior context, then listeners should be able to reliably distinguish among target ending times.
To evaluate the event-specific properties of expectancies, pattern context was varied. As in Experiments 1 and 2 , we manipulated the length of a sequence's recurrent melodic phrases (phrase context), holding constant true contrast when present ( \pm 1 beat). If the higher order time periods of phrases entirely determine expectancies, then the latter should systematically adjust to phrase length. This implies that phrase context will not significantly affect ending time judgments.

Two other aspects of pattern context that relate to event coherence were varied; one involves phrasing uniformity. In Experiments 1 and 2, standard sequences opened with a single deviant phrase and we assumed that this modest loss of phrase uniformity did not eliminate event coherence or significantly reduce expectancy generation. To verify this, phrase uniformity was varied in the present study such that some sequences had phrases of uniform length (even), while others contained a single deviant phrase (uneven); if this difference matters, then judgments should be less accurate and/or more variable in the latter conditions. The other variable that relates to coherence is a beat marker variable, which relates to the location of the silent period. The silent period lasted either one half of the total phrase duration (with $n$ markers), or less than this time span (with $n+1$ markers) (see Figure 3). We assume greater coherence in the former, because the silent period is more congruent with the nesting properties of a simple binary hierarchy. Thus, it is possible that people may render more accurate ending-time judgments in events containing fewer markers.

\section{Method}

\section{Design and Subjects}

The design was a $2 \times 3 \times 5 \times 2 \times 2$ mixed factorial. Counterbalance order was the single between-subjects variable. The four withinsubjects factors were target ending time (early, on time, late), phrase context (phrase length, $L=4,6,8,10,12$ beats), uniformity (even, uneven), and beat markers (few, more). Pattern instance (start pitches of $\mathrm{C} 4$ or $\mathrm{G4}$ ) was also a within-subjects factor, but was not analyzed.

Twenty-four subjects from an introductory psychology course at The Ohio State University volunteered for the experiment in exchange for course credit. In contrast to previous experiments, these subjects were musically unsophisticated. Twelve subjects were randomly assigned to each of the two counterbalance orders.

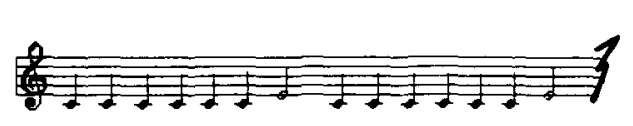

more $(n+1)$ markers

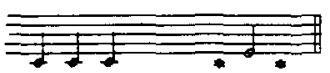

fewer (n) markers
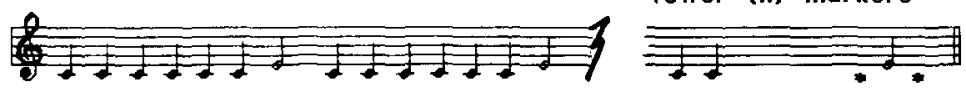

Figure 3. Two sequences of seven phrases (only two are shown, all lasting 8 beats, followed by a final phrase duration that includes either 2 (few) or 3 (more) beat markers prior to the silent period. Also shown is an on-time target (a half-note, o) and the locations of early and late targets (asterisks). 


\section{Stimulus Materials}

The four independent variables (phrase context, ending time, beat markers, uniformity) were factorially crossed to determine the structure of 60 basic patterns. Each pattern occurred once in each starting pitch, yielding a total of 120 sequences. All the patterns were modeled on the eight phrase sequences of Experiment 2, in which the modal lengths of the phrases varied $(\mathrm{L}=4,6,8,10$, or 12 beats); pitch and timing details of all the marking tones were identical as well. The primary structural differences with respect to sequences in Experiment 2 concerned target ending times and beat marker omissions.

The timing variable involved three different target ending times that corresponded to placing the final phrase marker either 1 beat early (i.e., $c=-1$ beat), 1 beat late (i.e., $c=1$ beat), or on time (i.e., $c=0$ ), given phrase length, L. In all cases, the beat marker variable dictated that the target tone immediately follow a silent period, which left either $n$ (fewer) or $n+1$ (more) tones opening the last phrase, where $n=0,1,2,3,4$ for $\mathrm{L}=4,6,8,10,12$, respectively. Thus, in on-time conditions, the silent period was either long $(\mathrm{L} \times 220) / 2$, or short $[(\mathrm{L} \times 220) / 2-220 \mathrm{msec}]$, respectively, for the fewer- and more-markers conditions (see Figure 3).

Uniformity refers to phrasing regularity associated with the pres ence (uneven) or absence (even) of an initial deviant phrase. Sixty patterns opened with a deviant 8-beat phrase (uneven) and 60 did not (even). (Note that for the $L=8$ condition, the initial 8 -beat phrase in the uneven condition was not technically "deviant.") Uneven and even sequences were comparable, respectively, to the standard and comparison sequences in Experiment 2.

\section{Apparatus}

All the stimulus patterns were constructed and generated using the MIDILAB software system (Todd et al., 1989). During each experimental session, the patterns were presented on line by a Yamaha TX81Z FM tone generator that was controlled by an IBMAT computer with a Roland MPU-401 MIDI interface unit. Tone sequences were amplified by a Kenwood KR-A4010 receiver and played over AKG k240 headphones at a comfortable listening level. All the tones were generated with a Pan Floot timbre (i.e., flutelike).

\section{Procedure}

Recorded instructions informed the subjects that they were to judge the timing of the final target tone relative to the pattern's preceding context, using a 15-point scale. They indicated whether the target seemed on time, early, or late by circling a number between -7 (very early), 0 (on time), and +7 (very late); use of the entire range was stressed.

The subjects removed their watches prior to the experiment and were tested in groups of 2-5 individuals. After 3 practice trials, they received two blocks of 60 experimental trials separated by a brief rest. Each experimental session lasted about 55 min.

\section{Results and Discussion}

Mean ratings were collapsed over pattern instance (a nonrelevant variable) as well as uniformity and counterbalance order (both statistically nonsignificant variables). Figure 4 presents mean ratings as a function of end timing, phrase context, and beat markers.

The most important findings concern the ending-time variable. First, the listeners were relatively good at judging "when" a target was on the mark or not $[F(2,44)=$ $\left.45.63, M S_{e}=12.52, p<.0001\right]$. Although they generally judged the final tone as being slightly early (i.e., a mean rating of -0.321 instead of 0.0 ), this bias was small compared with the large and systematic differences due to target timing. Post hoc Scheffé analyses indicated that the listeners significantly distinguished among all three conditions, in the following order: early $<$ on time $<$ late (see Figure 4). Second, this pattern of findings held across all phrase context conditions in that the listeners consistently gave early targets the lowest ratings and late targets the highest ratings. Thus, in spite of the fact that the absolute length of the silence differed both within and between different context conditions, the listeners nonetheless did fairly well in distinguishing among appropriate and inappropriate ending times. Both of these findings are consistent with the idea that expectancies about ending times are temporally sustained attentional extrapolations.

Next, we consider the event-specific properties of expectancies as they relate to both pattern context and the variables of phrase context, uniformity, and beat markers. If expectancies are determined entirely by phrase durations, then ideally we would expect no significant effects of phrase context, because the listeners would always recognize the correct and incorrect target times (with fixed contrast) relative to the prevailing phrase duration. But phrase length did affect ratings $\left[F(4,88)=11.06, M S_{\mathrm{c}}=\right.$ $16.43, p<.0001]$. Listeners are likely to judge targets as being "late" with short phrase structure and "early" with long phrase structures, revealing a phrase-based range effect. This was most evident in the on-time ratings (Figure 4) in which the listeners were most accurate with medium-length phrases. Overall, post hoc Scheffé analyses indicated several significant pairwise comparisons $(p<.01): 4$ versus 10 beats, 4 versus 12 beats, and 6 versus 12 beats.

In addition, although temporal contrast was held constant ( \pm 1 beat) across different phrasings, the listeners' sensitivity to it differed as a function of phrase length, leading to an interaction of phrase context with ending time $\left[F(8,176)=4.82, M S_{\mathrm{e}}=2.00, p<.0001\right]$. Distinctions between early, on-time, and late targets were generally preserved across different phrase lengths, but the ratings revealed that a 1-beat contrast was most noticeable when $L=4$ beats and least noticeable when $L=$ 6 or 12 beats. At least with respect to the 4- and 12-beat phrasings, these findings make sense if we assume that the listeners were responding not to the absolute magnitude of a contrast, but to its relative magnitude in the session. That is, a 1-beat change is more noticeable when it occurs relative to an expectancy for a short phrase than for a long one.

Of the remaining two variables, uniformity and beat markers, only the latter influenced performance. Because no statistically reliable differences in either means or variances related to even versus uneven phrasing, we can conclude that the small reductions in coherence associated with inclusion of an initial deviant phrase, as in the standard sequences of Experiments 1 and 2, did not substantially weaken expectancies.

The beat marker variable had a marginally significant influence on performance. As predicted, the listeners were more accurate with the patterns containing longer silences 

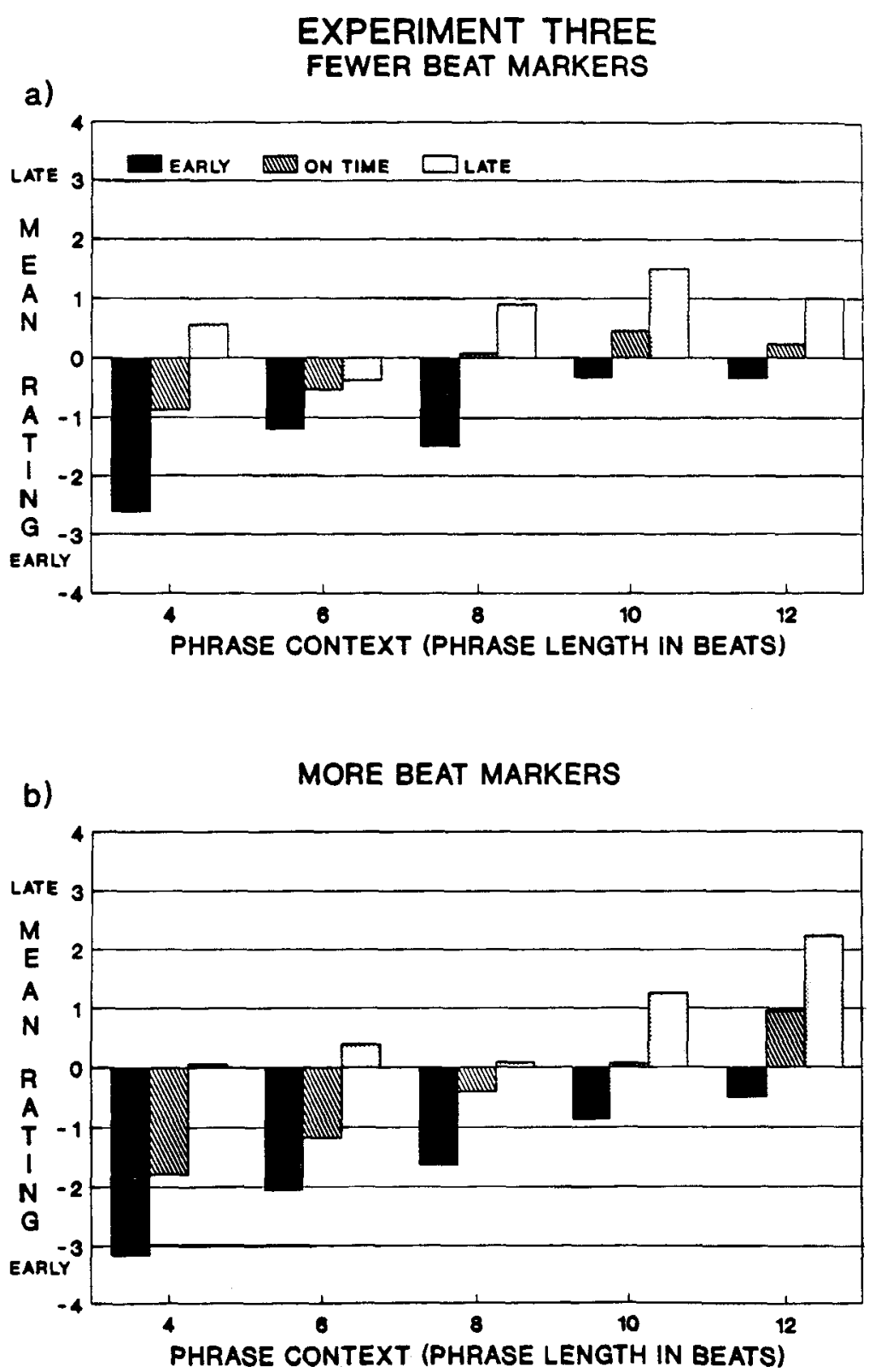

Figure 4. Timing ratings as a function of beat marker conditions, phrase length, and ending time in Experiment 3. Positive ratings indicate that the listeners judged the ending as "late" and negative ratings indicate "early" judgments. A zero rating indicates an "on-time" judgment.

(i.e., fewer beat markers) $\left[F(1,22)=4.24, M S_{\mathrm{e}}=4.46\right.$, $p<.0515]$. In general, the listeners produced higher ratings when the final phrase contained fewer marker tones, and because there was a general bias to judge on-time targets early; this translates into higher accuracy in these conditions. By some accounts, it seems contradictory that the patterns containing fewer marking tones and longer silences were judged more accurately. Conceivably, the patterns with more information should have produced greater accuracy; subjects can, in a sense, "hone in" on the correct ending time more precisely. This was not the case; the listeners were more likely in these conditions to judge the on-time endings of patterns with more markers as being early.

There were also interactions involving the beat marker variable. As shown in Figure 4, this variable significantly interacted with both phrase context $[F(4,88)=7.24$, $\left.M S_{\mathrm{e}}=2.46, p<.001\right]$ and phrase context and ending time $\left[F(8,176)=3.42, M S_{\mathrm{e}}=1.89, p<.0011\right]$. These interactions are best understood by considering the on-time target ratings. Notice that when fewer markers were present, the range effect due to phrase context centers on pat- 
terns with $\mathrm{L}=8$, (here, accuracy was greatest). However, with more markers, the range "center" shifts to patterns with $L=10$ (here, accuracy was greatest). The beat marker variable also affected the magnitude of the range effect in that the conditions with fewer markers, in which the listeners were more accurate, showed reduced distortions due to range extremes. An exception involves the sequences based on six-beat phrases, in which the listeners judged a late target as being early in the fewer-marker conditions; it is not clear why this was so. Nevertheless, generally with fewer beat markers: (1) greatest accuracy occurred with the mid-range eight-beat phrasings, and (2) bias was reduced in patterns with extreme phrase lengths.

One interpretation of these findings is that the listeners targeted their expectancies as a function of phrasing context in the same manner for both marker conditions, but that this strategy worked better with fewer beat markers. For example, assume that the listeners estimated the remaining silence by halving the duration of an abstracted phrase. If they then timed the onset of an attentional extrapolation on the basis of this estimate, from the last beat marker they would expect later endings in conditions with more markers than in ones with fewer markers. This would explain both the greater prevalence of early judgments of on-time endings and the shift of range center toward longer phrases in the conditions with more markers.

To sum up, Experiment 3 indicates that listeners can sustain attending over variable silent periods embedded within a final phrase so as to reliably distinguish among target ending times. Ratings of ending times are also affected by phrase durations within the preceding context and by the number of beats in the final phrase.

\section{EXPERIMENT 4}

In this experiment, we continued to explore eventspecific expectancies by relying on a temporal extrapolation task in which listeners overtly "completed" final time spans of incomplete sequences. In fact, the sequences were those used in Experiment 3, modified to exclude ending tones. Thus, whereas Experiment 3 held temporal contrast constant, Experiment 4 eliminated explicit contrasts, thus permitting assessment of "pure" expectancies.

The hypotheses of interest remain those posed in Experiment 3 relating to attentional extrapolations and their structural determinants. If expectancies reflect sustained event-specific attentional extrapolations, then this paradigm would permit an actual determination of ending time estimates vis-à-vis aspects of each event's context (e.g., phrasing variables and beat markers).

\section{Method}

\section{Design and Subjects}

The design was identical to that of Experiment 3, with the exception that ending time was not a factor. Thus, the variables were counterbalance order (between-subjects), and phrase context, uniformity, and beat markers (all within-subjects variables). Again, the pattern instance variable (start pitch of C4 or G4) was not analyzed.
Twenty-two musically naive subjects from an introductory psychology course at The Ohio State University volunteered for the experiment in exchange for course credit. Eleven of the subjects were randomly assigned to each of the two counterbalance orders.

\section{Stimulus Materials}

A total of 40 patterns, identical to those of Experiment 3 except for omitted target tones, were constructed. Each was played twice. The three independent variables (phrase context, uniformity, beat markers) were factorially balanced, so that the levels of each occurred equally often across all conditions.

\section{Apparatus and Procedure}

All the stimulus patterns were generated via MIDILAB, as in Experiment 3. Recorded instructions informed the subjects that they were to overtly "complete" each pattern. They were told to "press a button when you think the final tone should occur." Each produced time was automatically recorded via MIDILAB.

The subjects removed their watches prior to the experiment and were tested in groups of 2-5 individuals. After receiving 3 practice trials, they were presented with two blocks of 60 experimental trials separated by a brief rest. A full session lasted about $45 \mathrm{~min}$.

\section{Results and Discussion}

Figure 5 shows the produced ending times relative to implied target onset times as a function of phrase context and marker condition. Figure 6 shows the full completion times as a function of these variables plus the uniformity variable.

The data in Figures 5 and 6 suggest that people are fairly good at estimating the general neighborhood of a pattern's ending time. As Figure 5 indicates, the mean estimated ending time corresponds to a midway point in the duration of the final tone (i.e., $255 \mathrm{msec}$ after its implied onset time); however, modal response time was substantially closer to the implied target onset. These data imply that the listeners used phrasing to estimate target occurrence times. Confirming this, produced times, shown in Figure 6 , were strongly affected by phrase context $[F(4,80)$ $\left.=200.35, M S_{\mathrm{e}}=34,132, p<.00001\right]$. As predicted, the listeners' estimates of "when" an ending tone should occur increased as phrase length increased, suggesting that expectancies are sustained, event-specific attentional extrapolations that are determined, in part, by prominently marked phrasings.

Nevertheless, people's estimates of event endings are not entirely specific to phrasing properties. If this were so, then no range effects would be evident. However, as in Experiment 3, phrase context introduced range effects, as shown in Figure 5. Deviation times in this figure would be equivalent across phrase context if no range effects were present, but they are not; phrase context significantly modulated deviation times $\left[F(4,80)=16.28, M S_{e}=\right.$ $38,480, p<.0001]$. Relative to the mean of $255 \mathrm{msec}$, the listeners tended to produce longer response times (i.e., expect "later" endings) with shorter phrase lengths and shorter times (i.e., expect "earlier" endings) with longer phrase lengths, suggesting an absolute stimulus contraction bias (Poulton, 1989, pp. 155-166). This profile complements that found in Experiment 3 (see Figure 4). To- 


\section{EXPERIMENT FOUR DEVIATION TIMES}

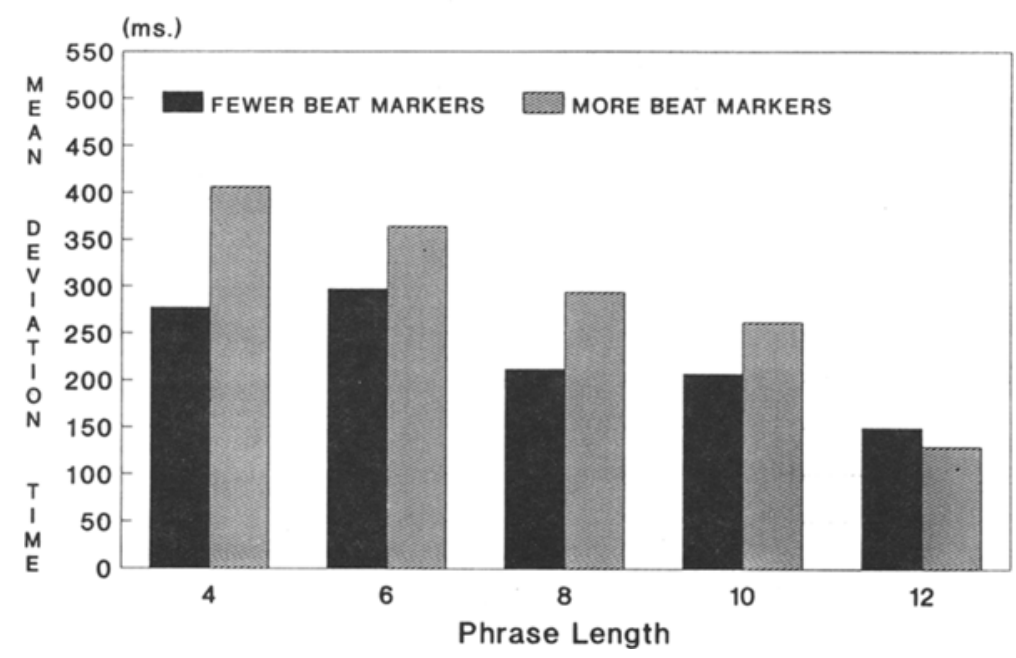

EXPERIMENT FOUR

PRODUCED ENDING TIMES

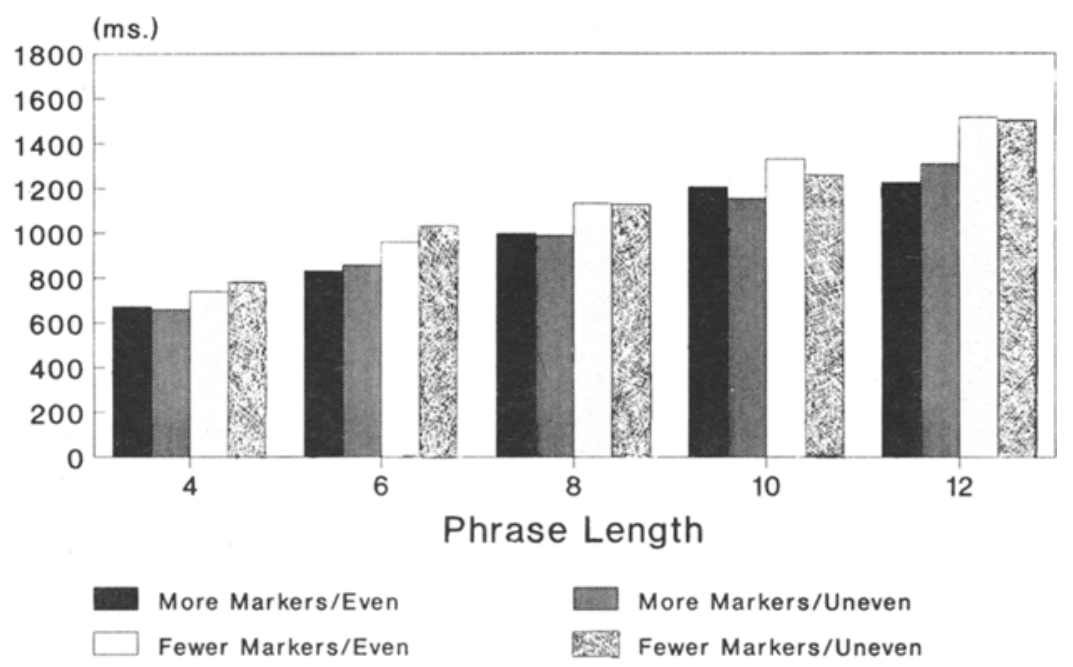


of phrase uniformity on produced times was modest, confined to an interaction with phrase context $[F(4,80)=4.95$, $\left.M S_{\mathrm{e}}=9,615, p<.005\right]$. The even phrasings stimulated slightly longer time estimates than the uneven ones, primarily in patterns having 10-beat phrases, whereas they stimulated shorter estimates with sequences having 6- and 12 beat phrases (Figure 6). Thus, when a shorter 8-beat phrase appeared amidst 10-beat phrases, the listeners shortened their final phrase estimates; but when this same deviant phrase appeared among 6-beat phrases, they produced somewhat longer estimates. Only the 12-beat case (in which the difference amounted to $24 \mathrm{msec}$ ) seems difficult to explain. For the most part, these are small time differences, with all conditions yielding ending-time estimates within the temporal neighborhood of the true implied endings.

Finally, the beat marker variable introduced a large effect on produced times $\left[F(1,20)=396.61, M S_{e}=8,515\right.$, $p<.0001]$. In general, the produced estimates of remaining times were longer with the more truncated patterns (i.e., the ones with fewer final beat markers), indicating that the listeners did flexibly adjust these estimates according to the prevailing context. The average difference between the two marker conditions was $185 \mathrm{msec}$, which is slightly less than their true difference in length in the final phrase. Because it was stressed that the listeners should estimate the time of occurrence of the final tone (although onset time was not specifically mentioned), we can also ask whether deviation times relative to true implied onset times of targets differed as a function of marker condition. They did. As shown in Figure 5, the fewermarkers conditions resulted in more accurate ending-time estimates (by this criterion) $\left[F(1,20)=23.70, M S_{\mathrm{e}}=\right.$ $24,582, p<.0001]$. These data are consistent with those of Experiment 3, which showed that the listeners' on-time target ratings were more accurate in the fewer-markers conditions.

Finally, this experiment was designed to determine the extent to which expectancies about ending times of incomplete phrases realize attentional extrapolations that are event specific; that is, determined by phrasing context (including phrase length) and beat markers. A final analysis of the produced ending-time estimates regresses these productions on phrase duration $(X=L \times 220)$ for each marker condition. The results of this analysis appear in Figure 7, along with an indication of the implied target onset times. They indicate that a strong linear relationship obtains between phrase duration and produced time spans in each marker. In each case, the linear relation between phrase duration and produced time estimates accounts for over $98 \%$ of the variance, in spite of variations due to range effects. The two marker conditions differed as a function of intercept (Figure 7a vs. 7b); this finding is consistent with the interpretation that the listeners in the conditions with fewer markers "waited" longer after the final marker tone before initiating a time estimate. On the whole, however, these data are consistent with the view that the listeners flexibly adjusted their extrapolations of estimated ending times as a function of phrase length and marker condition and that their strategy resulted in expected ending times that were, on the average, closer to the implied target onsets in the conditions in which the remaining time was exactly half the total phrase duration (i.e., the fewer-markers condition). In sum, these data are consistent with the idea that, in the absence of explicit temporal contrasts, listeners with modest musical training can sustain their attention over substantial silent periods to reveal event-specific expectancies.

\section{GENERAL DISCUSSION}

It appears that one song will "seem" longer than another of equivalent length when it violates real-time expectancies about "when" it should end; that is, when it ends later than an implied ending time. The key features of this phenomenon have to do with how the temporal information that fills a tune's interval unfolds in time. It is not simply the amount of information (tones, chunks, changes, phrases, etc.) that fills a time interval that determines judgments, but rather its temporal distribution. In Experiments 1 and 2, to-be-judged intervals were alike in both amount of information and total duration, and yet the listeners routinely judged them as differing in total duration. What mattered was "when" in time a single deviant phrase occurred; if it opened a standard pattern, it had negligible influence on time estimates, but if it closed a comparison sequence, it had marked effects. In this and related experiments (e.g., Boltz, 1991b; Jones \& Boltz, 1989), when information within judged intervals formed coherent patterns, over- and underestimations of identical durations depended on the temporal arrangement of information within these intervals. Two features of these arrangements involve the degree of coherence that determines the likelihood of future-oriented attending and the magnitude and direction of temporal contrast that affects the nature of time distortions.

In this regard, much of these data tap into our notoriously malleable sense of elapsed time in various everyday situations, where we find ourselves "caught up" in the flow of a conversation, basketball game, or symphony. Typically, the unfolding dynamic structure of such events impels us to continually anticipate their future course. In these contexts, when violations of event-induced expectancies occur, they create momentary surprises (temporal contrasts), and our claim is that these contrasts have the power to bias time judgments.

Because beginning and ending times delimit an event's total duration, final contrasts that seem to imply a changed ending can distort judgments of the whole event's duration. This was evident in Experiments 1 and 2, in which comparative judgments were affected not only by the direction of a final temporal contrast, but also by its mag- 

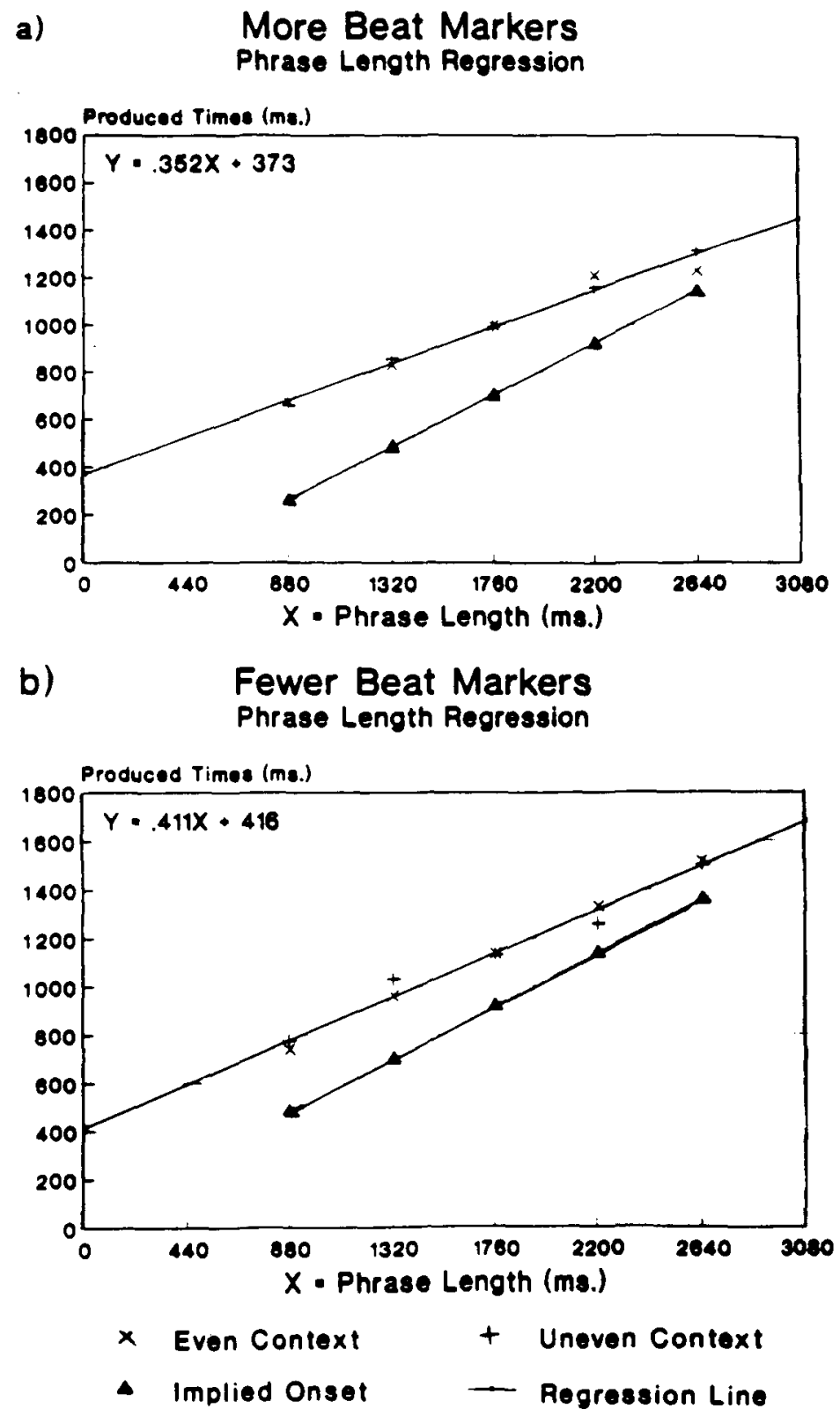

Figure 7. Produced times as a function of phrase length for the more-beatmarkers condition (a) and the fewer-beat-markers condition (b). Data for even and uneven uniformity conditions are shown in each marker condition. Also shown is the implied (correct) onset time for a target ending tone.

nitude. The finding that contrast magnitude affects time estimates is difficult for some contrast models. In Kristofferson's $(1977,1984)$ contrast model, time discrimination results from an ordinal comparison of an internal time period, corresponding to a modal duration within an experimental session, with a given observed duration. This implies that, regardless of the magnitude of contrast between expected and observed intervals, the one that ends first is judged as being "shorter." This model does not appear to extend to the rating tasks used in the first two experiments, in which contrast magnitude did affect comparative time judgments.

Both direction and magnitude of a contrast depend on expectancies, and expectancies were the focus of Experiments 3 and 4 . These studies found support for the expectancy/contrast model's predictions that expectancies are timed attentional extrapolations that are event specific (Jones, 1976, 1981). In fact, the data from all four experiments are consistent with the hypothesis that coherent phrasing determines expectancies in that they show 
that listeners expect longer (or shorter) final phrases in patterns with longer (or shorter) phrasing contexts. However, Experiment 3 most clearly illustrates that people's ratings of "when" target tones should occur are reliable and shift systematically with phrase context. Converging evidence comes from Experiment 4, in which the listeners had to temporally complete incomplete patterns. Excellent fits of the data to regression equations incorporating phrase length as a determinant of expected ending times suggest that people sustain attending over substantial silences in a lawful, event-specific way that depends on contextual properties such as phrase structure. Finally, manipulations of the beat marker variable in Experiments 3 and 4 provide suggestive, but not conclusive, support for the idea that listeners' expectancies are sensitive to time relationships that conform to binary time hierarchies.

Because expectancies are cast as timed attentional extrapolations, this view differs from a more conventional approach that casts an expectancy in terms of a probability measure that is not conditionalized on real time. According to the latter account, the most probable tone frequency (e.g., here, that of the lower pitch) determines what is expected. Unfortunately, this tells little about "when" a person anticipates that pitch; more importantly, it says nothing about how listeners might anticipate "when" a less frequent pitch occurs (i.e., the phrase marker tone).

The data from all of these studies speak to the issue of generality. For example, the findings in Experiments 1 and 2 generalized to patterns with different contextual properties (e.g., filler patterns). And these data converge with other findings, using true musical events, which show that parallel effects of contrast magnitude and direction can result from manipulations of final phrase duration (Boltz, 1991b; Jones \& Boltz, 1989).

Perhaps a more interesting aspect of the generality issue concerns domain specificity. To what degree are the constructs of expectancy and temporal contrast specific to the musical domain and to listeners especially familiar with this domain? Experiments 3 and 4 indicate that both expectancies (Experiment 4) and temporal contrast (Experiment 3) are not confined to listeners who are especially familiar with musical principles. In these studies, ordinary listeners showed reliable sensitivities to contextual phrasings as well as to contrast manipulations. But with regard to the larger issue concerning nonmusical domains, we might ask to what degree do such findings generalize to duration judgments about nonmusical events? The expectancy/contrast model focuses on temporal structure, and so it provides a theoretical rationale for predicting generality across other domains and, indeed, modalities. Patterning in time is ubiquitous. When temporal patterning is coherent, there is empirical evidence that expectancies and expectancy violations (contrast) arising from manipulations of speech timing properties affect time judgments of spoken utterances (Lehiste, 1975a, 1975b). Similarly, de Wied, Tan, and Frijda (1992) have found that systematic variations in the expected rate of action can influence the judged duration of filmed narratives. But temporal expectancies and contrast can be induced in other ways. Boltz (1992c) recently examined the impact of expectancies arising from consensual agreement about the amount of elapsed time or activity required in a task prior to its completion. In one experiment, subjects were led to expect a given number of future trials, whereas in a second experiment, different expectancies about the duration of a waiting period were induced. In both contexts, confirmation/violation of expectancies exerted a significant impact on retrospective duration judgments. When the subjects received more/fewer trials than expected or waited for durations longer/shorter than anticipated, they respectively over- and underestimated the same total durations. By contrast, time judgments of confirmed intervals were quite accurate. These results show that the expectancy construct has generality beyond the immediate domain. Finally, of course, a thorough evaluation of domain generality, by definition, entails substantially more research along these lines.

Another aspect of the generality question concerns temporal coherence. Because the expectancy/contrast model links time estimation to temporal coherence and attending mode, it does not imply that temporal contrast effects are general across a continuum of coherence. Time estimation effects due to temporal contrast are specific to the future-oriented attending mode. In the present research, we used coherent events in order to focus on this mode. However, many events are not regular or coherent, and analytic attending occurs in these (Jones \& Boltz, 1989). In contrast to future-oriented attending, the analytic attending mode involves focusing over time levels of fine details, and the resulting time estimates depend on the relative pace and number of changes at these levels and not upon expectancies and temporal contrast. The most direct evidence for this distinction comes from Boltz (1991b), who asked subjects to compare the duration of two melodies in which the timing of higher order phrase endings was temporally coherent, but lower order contour changes among adjacent pitches (i.e., their "ups and downs") were not. When the subjects attentionally tracked the higher level of structure (i.e., future-oriented attending), their time estimations were determined by structurally based expectancies and temporal contrast. However, when they tracked the irregular lower level of structure (analytic attending), their judgments were determined by the number of contour changes, with more changes producing longer time estimates. In short, the listeners' comparative time estimates of the same melody were either over- or underestimations, depending upon attending mode and temporal coherence.

The distinction between coherent and noncoherent events is a basic one in this model and it holds across different judgment tasks (absolute vs. comparative). In absolute judgments, expectancies about the duration of even a single event can arise, provided its structure is coherent (e.g., Experiments 3 and 4); in comparative time judgments (Experiments 1 and 2), expectancies about a com- 
parison sequence depend on coherence of both standard and comparison sequences (see Jones \& Boltz, 1989).

Does all of this mean that whenever a coherent event appears, event-specific expectancies are mandatory attentional responses? The most likely answer to this question is: "Not exactly." In the present tasks, the listeners were encouraged to use context and received no feedback regarding time estimation errors. We cannot determine the degree to which people would or could actually ignore phrasing context prior to a final phrase in other settings. However, it is clear from the data of Experiment 2 that the listeners did not ignore context in the current tasks. But would the same pattern of results occur, for example, in a retrospective task in which listeners did not know in advance that time estimates would be required? The study by Boltz (1992c) suggests that it may. However, goals, instructions, and other task parameters inevitably affect how people attend (e.g., see discussion in Jones $\&$ Boltz, 1989). Therefore, we cannot rule out the possibility that a listener can be encouraged to ignore coherent phrasings. In this sense, the influence of a particular level of coherent contextual structure is not mandatory. Nevertheless, in cases in which context carries taskrelevant information that is coherently arranged in time, we claim that its structural properties are naturally compelling ones that do determine expectancies.

Finally, the present data qualify assumptions of the expectancy/contrast model in two respects. First, with regard to the issue of the event specificity of expectancies, the finding of range effects somewhat weakens this claim. If expectancies are driven only by the context of a specific event, then no range effects should appear. That range effects are present lends some support to Kristofferson's hypothesis that listeners' expectancies are based on various durations experienced over an entire session (Kristofferson, 1977, 1984). The influence of extreme stimuli (here, $L$ of 4 and 12 ) has been documented in many other tasks (e.g., Parducci, 1984; Poulton, 1989). In addition, with respect to temporal variables, session effects associated with tempo and/or rhythm have been previously reported (e.g., Jones, Kidd, \& Wetzel, 1981; Kidd, Boltz, \& Jones, 1984). However, in the present case, the range effects were small compared with those associated with manipulations of contrast (Experiments 1-3) and absolute phrase length (Experiment 4). Indeed, in the regression analyses of Experiment 4, which ignored range effects, over $98 \%$ of the variance was explained in terms of phrase-specific expectancies. Thus, while the term event-specific, taken to mean a single pattern, may prove too restrictive, the present data suggest that phrasing structure is a dominant influence on expectancies.

A second qualification concerns temporal contrast. The data of Experiment 3 suggest that listeners may rely more on relative contrast magnitude than absolute differences between expected and observed endings. According to the expectancy/contrast model, the 1-beat contrast in that study should have the same impact on ratings, regardless of phrase context. This assumption is clearly wrong. A difference of 1 beat has a greater impact on patterns with 4beat phrases than on those with 12-beat phrases. Thus, instead of $c^{\prime}=\mathrm{O}-\mathrm{E}$, we must assume that $c^{\prime}=(\mathrm{O}-\mathrm{E}) / \mathrm{E}$.

In summary, people respond to patterned arrangements of tones by generating expectancies about "when" these events will end. Expectancies involve real-time attentional extrapolations, which tend to mirror the phrasing properties of the events themselves. Expectancy violations, in the form of relative temporal contrast, affect judgments about comparative durations of such events.

\section{REFERENCES}

Allan, L. G. (1979). The perception of time. Perception \& Psychophysics, 26, 340-354.

Avant, L., \& Lyman, P. (1975). Stimulus familiarity modifies perceived duration in prerecognition visual processing. Journal of $E x$ perimental Psychology: Human Perception \& Performance, 1 , 205-213.

Block, R. (1979). Time and consciousness. In G. Underwood \& R. Stevens (Eds.), Aspects of consciousness: Vol. I. Psychological issues (pp. 179-217). London: Academic Press.

Block, R. (1985). Contextual coding in memory: Studies of remembered duration. In J. A. Michon \& J. L. Jackson (Eds.), Time, mind, and behavior (pp. 169-178). Heidelberg: Springer-Verlag.

BLOCK, R. (1989). Experiencing and remembering time: Affordances, context, and cognition. In I. Levin \& D. Zakay (Eds.), Time and human cognition: A life-span perspective (pp. 333-363). Amsterdam: North-Holland.

Boltz, M. (1985). An expectancy model of judged duration: An ecological perspective. Unpublished doctoral dissertation, The Ohio State University

BoLtz, M. (1989a). Perceiving the end: Effects of tonal relationships on melodic completion. Journal of Experimental Psychology: Human Perception \& Performance, 15, 749-761.

Boltz, M. (1989b). Time judgments of musical endings: Effects of expectancies on the "filled interval effect." Perception \& Psychophysics, 46, 409-418.

BoLTz, M. (1991a). Some structural determinants of melody recall. Memory \& Cognition, 19, 239-251.

BoLtz, M. (1991b). Time estimation and attentional perspective. Perception \& Psychophysics, 49, 422-433.

BoLTZ, M. (1992a). The remembering of auditory event durations. Journal of Experimental Psychology: Learning, Memory, \& Cognition, 18, 938-956

BoLtz, M. (1992b). Temporal accent structure and the remembering of filmed narratives. Joumal of Experimental Psychology: Human Perception \& Performance, 18, 90-105.

BoLTZ, M. (1992c). Time estimation and expectancies. Manuscript submitted for publication.

Boltz, M., \& JoNEs, M. (1986). Does rule recursion make melodies easier to reproduce? If not, what does? Cognitive Psychology, 18, 389-431.

Brown, S. W. (1985). Time perception and attention: The effects of prospective versus retrospective paradigms and task demands on perceived duration. Perception \& Psychophysics, 38, 115-124.

BuRNSIDE, W. (1971). Judgment of short time intervals while performing mathematical tasks. Perception \& Psychophysics, 9, 404-406.

Clarke, E. F., Krumhansl., C. L. (1990). Perceiving musical time. Music Perception, 7, 213-251.

COOPER, W., \& SORENSON, J. (1977). Fundamental frequency contours at syntactic boundaries. Journal of the Acoustical Society of America, 62, 683-692

Cutting, J. (1981). Coding theory adapted to gait perception. Journal of Experimental Psychology: Human Perception \& Performance, 7 , $71-87$. 
Devane, J. (1974). Word characteristics and judged duration for two response sequences. Perceptual \& Molor Skills, 38, 525-526.

DE WIED, M., TAN, E., FRIJAA, N. (1992). Duration experiences under conditions of suspense in films. In F. Macar, V. Pouthas, \& W. Fried man (Eds.), Time, action, and cognition (pp. 325-336). Dordrecht: Kluwer Academic Publishers.

Fraisse, P. (1978). Time and rhythm perception. In E. Carterette \& W. Friedman (Eds.), Handbook of perception (Vol. 8, pp. 203-254). New York: Academic Press.

Fraisse, P. (1984). Perception and estimation of time. Annual Review of Psychology, 35, 1-36.

FraISSE, P. (1987). Cognition of time in human activity. In G. d'Ydewalle \& W. Lens (Eds.), Cognition in human motivation and learning (pp. 233-259). Hillsdale, NJ: Erlbaum.

FRANKENHAUSER, M. (1959). Estimation of time: An experimental study. Stockholm: Almquist \& Wiksell.

GARNER, W. (1951). The accuracy of counting repeated short tones Journal of Experimental Psychology, 41, 310-316.

GENTNER, D. (1987). Timing of skilled motor performance: Tests of the proportional duration model. Psychological Review, 94, 255-276.

GiBson, J. (1979). The ecological approach to visual perception. Boston: Houghton Mifflin.

Grosjean, F., Grosjean, L., \& Lane, H. (1979). The patterns of silence: Performance structures in sentence production. Cognitive Psychology, 11, 58-81.

Hicks, R., Miller, G., \& Kinsbourne, M. (1976). Prospective and retrospective judgments of time as a function of amount of information processed. American Journal of Psychology, 89, 719-730.

JoNes, M. R. (1976). Time, our lost dimension: Toward a new theory of perception, attention, and memory. Psychological Review, 83 , 323-335.

JONES, M.R. (1981). Only time can tell: On the topology of mental space and time. Critical Inquiry, 7, 557-576.

JONES, M.R. (1987). Dynamic pattern structure in music: Recent theory and research. Perception \& Psychophysics, 41, 621-634.

JONES, M. R., \& BoLTz, M. (1989). Dynamic attending and responses to time. Psychological Review, 96, 459-491.

JonES, M. R., KIDD, G., \& WETZEL, R. (1981). Evidence for rhythmic attention. Journal of Experimental Psychology: Human Perception \& Performance, 7, 1059-1073.

Jones, M. R., \& Ralston, J. T. (1991). Some influences of accent structure on melody recognition. Memory \& Cognition, 19, 8-20.

KIDD, G., BolTZ, M., \& JONES, M. R. (1984). Some effects of thythmic context on melody recognition. American Journal of Psychology, 97, 153-173.

Kramer, J. (1982). Beginnings and endings in Western art music. Canadian University Music Review, 3, 1-14

KRISTOFferson, A. B. (1977). A real-time criterion theory of duration discrimination. Perception \& Psychophysics, 21, 105-117.

Kristofferson, A. B. (1984). Quantal and deterministic timing in human duration discrimination. Annals of the New York Academy of Sciences, 423, 3-15.

LEHISTE, I. (1975a, August). The perception of duration within sequences of four intervals. Paper presented at the Eighth International Congress of Phonetic Sciences, Leeds, England.

Lehiste, I. (1975b). The phonetic structure of paragraphs. In A. Cohen \& S. G. Nootenboom (Eds.), Structure and process in speech perception (pp. 195-206). New York: Springer-Verlag.

LERDAHL, F., JACKENDOFF, R. (1983). A generative theory of tonal music. Cambridge, MA: MIT Press.

MANdler, J., \& Johnson, N. (1977). Remembrance of things parsed: Story structure and recall. Cognitive Psychology, 9, 111-151.

MASSARo, D. (1976). Perceiving and counting sounds. Journal of Ex- perimental Psychology: Human Perception \& Performance, 2, 337-346.

MCCLAIN, L. (1983). Interval estimation: Effect of processing demands on prospective and retrospective reports. Perception \& Psychophysics, 34, $185-189$.

Meyer, L. (1956). Emotion and meaning in music. Chicago: University of Chicago Press.

NEWTSON, D. (1973). Attribution and the unit of perception of ongoing behavior. Journal of Personality \& Social Psychology, 28, 28-38.

Ornstein, R. (1969). On the experience of time. Baltimore, MD: Penguin Books.

Palmer, C. (1989). Mapping musical thought to musical performance. Joumal of Experimental Psychology: Human Perception \& Performance, 15, 331-346.

Parducci, A. (1984). Perceptual and judgmental relativity. In V. Sarris \& A. Parducci (Eds.), Perspectives in psychological experimentation: Toward the year 2000 (pp. 135-150). Hillsdale, NJ: Erlbaum

Pierson, K. (1976). Control of walking. Scientific American, 235, 72-86. Piston, W. (1987). Harmony (5th ed.). New York: Norton.

Poulton, E. C. (1989). Bias in quantifying judgments. Hillsdale, NJ: Erlbaum

Poynter, W. D. (1983). Duration judgment and the segmentation of experience. Memory \& Cognition, 11, 77-82.

SCHIFFMAN, H., \& BOBKo, D. (1974). Effects of stimulus complexity on the perception of brief temporal intervals. Journal of Experimental Psychology, 103, 156-159.

ScotT, D. (1982). Duration as a cue to the perception of a phrase boundary. Joumal of the Acoustical Society of America, 71, 996-1007.

Thomas, E. A. C., \& Weaver, W. B. (1975). Cognitive processing and time perception. Perception \& Psychophysics, 17, 363-367.

ToDD, N. (1985). A model of expressive timing in tonal music. Music Perception, 3, 33-58.

TodD, R., Boltz, M., \& Jones, M.R. (1989). The MIDILAB auditory research system. Psychomusicology, 8, 83-96.

UNDERWOOD, G., \& SWAIN, R. (1973). Selectivity of attention and the perception of duration. Perception, 2, 101-105.

ZAKAY, D. (1989). Subjective time and attentional resource allocation: An integrated model of time estimation. In I. Levin \& D. Zakay (Eds.), Time and human cognition: A life-span perspective (pp. 365397). Amsterdam: North-Holland.

ZakaY, D., NitZan, D., \& Glicksohn, J. (1983). The influence of task difficulty and external tempo on subjective time estimation. Perception \& Psychophysics, 34, 451-456.

\section{NOTES}

1. In much of Western music, the endings of melodic phrases within a tune are marked by members of the tonic triad chord. These consist of the tonic, mediant, and dominant pitches within a key; they correspond, respectively, to the first, third, and fifth scale steps of a melody's underlying diatonic scale (e.g., the pitches C, E, and G for the C Major diatonic scale). The ending of a melody itself is often marked by the presence of a leading tone (seventh scale degree) to tonic progression (Kramer, 1982; Piston, 1987). As event endings, these pitch markers are usually rated as being more complete and convey a sense of resolution to the listener (Boltz, 1989a).

2. Event specific is used here to indicate relationships that constrain the pattern structure of a temporal event. It is not inconsistent with the term event, as used, for example, by Gibson (1979).

(Manuscript received April 20, 1992; revision accepted for publication March 11, 1993.) 